\section{Fast-slow dynamical approximation of forced impact systems near periodic solutions}

\author{
Flaviano Battelli ${ }^{1}$ and Michal Fečkan ${ }^{2 *}$
}

${ }^{\text {CCorrespondence: }}$
Michal.Feckan@fmph.uniba.sk
${ }^{2}$ Department of Mathematical
Analysis and Numerical
Mathematics, Faculty of
Mathematics, Physics and
Informatics, Comenius University,
Mlynská dolina, Bratislava, 842 48,
Slovakia
Full list of author information is
available at the end of the article

${ }^{*}$ Correspondence:

Michal.Feckan@fmph.uniba.sk Department of Mathematical

merical

Informatics, Comenius University,

Full list of author information is

available at the end of the article

\begin{abstract}
We approximate impact systems in arbitrary finite dimensions with fast-slow dynamics represented by regular ODE on one side of the impact manifold and singular ODE on the other. Lyapunov-Schmidt method leading to Poincaré-Melnikov function is applied to study bifurcations of periodic solutions. Several examples are presented as illustrations of abstract theory.
\end{abstract}

MSC: 34C23; 34C25; 37G15; 70K50

Keywords: fast-slow dynamics; impact systems; bifurcations; periodic solutions; Poincaré-Melnikov method

\section{Introduction}

Non-smooth differential equations when the vector field is only piecewise smooth, occur in various situations: in mechanical systems with dry frictions or with impacts, in control theory, electronics, economics, medicine and biology (see [1-6] for more references). One way of studying non-smooth systems is a regularization process consisting on approximation of the discontinuous vector field by a one-parametric family of smooth vector fields, which is called a regularization of the discontinuous one. The main problem then is to preserve certain dynamical properties of the original one to the regularized system. According to our knowledge, the regularization method has been mostly used to differential equations with non-smooth nonlinearities, like dry friction nonlinearity (see [7] and a survey paper [8]). As it is shown in [7, 8], the regularization process is closely connected to a geometric singular perturbation theory $[9,10]$. On the other hand, it is argued in [11] that a harmonic oscillator with a jumping non-linearity with the force field nearly infinite in one side is a better model for describing the bouncing ball, rather then its limit version for an impact oscillator. This approach is used also in [12] when an impact oscillator is approximated by a one-parametric family of singularly perturbed differential equations, but as discussed in [12], the geometric singular perturbation theory does not apply.

In this paper, we continue in a spirit of [12] as follows. Let $\Omega \subset \mathbb{R}^{n}$ be an open subset and $G: \Omega \rightarrow \mathbb{R}$ a $C^{2}$-function, such that $G^{\prime}(x) \neq 0$ for any $x \in S:=\{x \in \Omega \mid G(x)=0\} \subset \Omega$. Then $S$ is a smooth hyper-surface of $\Omega$ that we call impact manifold, (or hyper-surface). We set $\Omega_{ \pm}=\{x \in \Omega \mid \pm G(x)>0\}$ and consider the following regular-singular perturbed system:

$$
\begin{cases}\varepsilon \dot{x}=f_{+}(x)+\varepsilon g_{+}(t, x, \varepsilon) & \text { for } x \in \Omega_{+}, \\ \dot{x}=f_{-}(x)+\varepsilon g_{-}(t, x, \varepsilon) & \text { for } x \in \Omega_{-}\end{cases}
$$

\section{照 Springer}

(c) 2013 Battelli and Fečkan; licensee Springer. This is an Open Access article distributed under the terms of the Creative Commons Attribution License (http://creativecommons.org/licenses/by/2.0), which permits unrestricted use, distribution, and reproduction in any medium, provided the original work is properly cited. 
for $\varepsilon>0$ small. We assume that the system

$$
\begin{cases}\dot{x}=f_{+}(x) & \text { for } x \in \Omega_{+}, \\ \dot{x}=f_{-}(x) & \text { for } x \in \Omega_{-}\end{cases}
$$

has a continuous periodic solution $q(t)$ crossing transversally the impact manifold $S$, given by

$$
q(t)= \begin{cases}q_{-}(t) \in \Omega_{-} & \text {for }-T_{-}^{0}<t<0, \\ q_{+}(t) \in \Omega_{+} & \text {for } 0<t<T_{+}^{0}\end{cases}
$$

and $q_{-}(0)=q_{+}(0) \in S, q_{-}\left(-T_{-}^{0}\right)=q_{+}\left(T_{+}^{0}\right) \in S$. By transversal crossing, we mean that

$$
G^{\prime}\left(q\left( \pm T_{ \pm}^{0}\right)\right) \dot{q}_{ \pm}\left( \pm T_{ \pm}^{0}\right)<0<G^{\prime}(q(0)) \dot{q}_{ \pm}(0)
$$

We set $T_{\varepsilon}:=T_{-}^{0}+\varepsilon T_{+}^{0}$ and assume that $g_{ \pm}(t, x, \varepsilon)$ are $T_{\varepsilon}$-periodic in $t$.

Transversal crossing implies that (1.2) has a family of continuous solutions $q(t, \alpha), \alpha \in$ (an open neighborhood $I_{0}$ of $0 \in \mathbb{R}^{n-1}$ crossing transversally the impact manifold $S$, given by

$$
q(t, \alpha)= \begin{cases}q_{-}(t, \alpha) \in \Omega_{-} & \text {for }-T_{-}(\alpha)<t<0, \\ q_{+}(t, \alpha) \in \Omega_{+} & \text {for } 0<t<T_{+}(\alpha),\end{cases}
$$

where $q_{-}(0, \alpha)=q_{+}(0, \alpha) \in S, q_{-}\left(-T_{-}(\alpha), \alpha\right), q_{+}\left(T_{+}(\alpha), \alpha\right) \in S$, and $q_{ \pm}(t, 0)=q_{ \pm}(t)$ and $T_{ \pm}(0)=T_{ \pm}^{0}$. Moreover, $T_{ \pm}(\alpha)$ is $C^{2}$ in $\alpha$, and the maps $\alpha \mapsto q(0, \alpha)$ and $\alpha \mapsto q\left( \pm T_{ \pm}(\alpha), \alpha\right)$ give smooth $\left(C^{2}\right)$ parameterizations of the manifold $S$ in small neighborhoods $U_{0}$ of $q(0)$ and $U_{ \pm}$of $q\left(T_{+}^{0}\right)=q\left(-T_{-}^{0}\right)$. Then the map $R: U_{0} \cap S \rightarrow U_{+} \cap S, q(0, \alpha) \mapsto q_{+}\left(T_{+}(\alpha), \alpha\right)$ is $C^{2}$-smooth. In this paper, we study the problem of existence of a $T_{\varepsilon}$-periodic solution of the singular problem (1.1) in a neighborhood of the set

$$
\left\{q_{-}(t) \mid t \in\left[-T_{-}^{0}, 0\right]\right\} \cup\left\{q_{+}(t) \mid t \in\left[0, T_{+}^{0}\right]\right\} .
$$

As a matter of fact, in the time interval $\left[0, \varepsilon T_{+}^{0}\right]$, resp. $\left[-T_{-}^{0}, 0\right]$, the periodic solutions will stay close to $q_{+}\left(\varepsilon^{-1} t\right)$, resp. to $q_{-}(t)$, and hence it will pass from the point of $S$ near $q(0)$ to the point of $S$ near $q_{+}\left(T_{+}^{0}\right)$ in a very short time (of the size of $\left.\varepsilon T_{+}^{0}\right)$. So, we may say that the behavior of the periodic solutions of (1.1) in the interval $\left[-T_{-}^{0}, \varepsilon T_{+}^{0}\right]$ is quite well simulated by the solution of the perturbed impact system

$$
\begin{aligned}
& \dot{x}=f_{-}(x), \\
& R\left(q_{-}(0, \alpha)\right)=q_{+}\left(T_{+}(\alpha), \alpha\right) .
\end{aligned}
$$

It is now clear that our study has been mostly motivated by the paper [12], where a similar problem on planar perturbed harmonic oscillators is studied. However arguments in [12] are mainly based on averaging methods whereas, in this paper, we investigate a general higher-dimensional singular equation such as (1.1) by using the Lyapunov-Schmidt reduction. We focus on the existence of periodic solutions and do not check their local 
asymptotic properties as, for example, stability or hyperbolicity. This could be also done by following our approach but we do not go into detail in this paper.

Our results (see Theorems 3.1 and 5.1) state that if a certain Poincaré-Melnikov-like function has a simple zero then the above problem has an affirmative answer. The proof of this fact is accomplished in several steps. In Section 2, we show, for any $\alpha$ in a neighborhood of $\alpha=0$, the existence of a unique continuous solution $x(t)=x(t, \alpha, \varepsilon)$ of (1.1) near the set $\left\{q(t, \alpha) \mid t \in\left[-T_{-}^{0}, T_{+}^{0}\right]\right\}$ which is defined in $\left[-T_{-}+\tau, \varepsilon T_{+}+\tau\right], T_{ \pm} \simeq T_{ \pm}^{0}$ and such that $x(\tau)=q(0, \alpha)$, for some $\tau$, and $x\left(-T_{-}+\tau, \alpha, \varepsilon\right), x\left(\varepsilon T_{+}+\tau, \alpha, \varepsilon\right)$ belong to $U_{ \pm} \cap S$. Moreover, $\alpha \mapsto x\left(-T_{-}+\tau, \alpha, \varepsilon\right)$ and $\alpha \mapsto x\left(\varepsilon T_{+}+\tau, \alpha, \varepsilon\right)$ are $C^{2}$ close to $q_{ \pm}\left( \pm T_{ \pm}(\alpha), \alpha\right)$ and then $\alpha \mapsto x\left(-T_{-}+\tau, \alpha, \varepsilon\right)$ and $\alpha \mapsto x\left(\varepsilon T_{+}+\tau, \alpha, \varepsilon\right)$ give $C^{2}$ parameterizations of $S$ in neighborhoods of $q_{ \pm}\left( \pm T_{-}(\alpha), \alpha\right)$. Hence, $x\left(-T_{-}+\tau, \alpha, \varepsilon\right) \mapsto x\left(\varepsilon T_{+}+\tau, \alpha, \varepsilon\right)$ gives a Poincaré-like map and a $\left(T_{-}^{0}+\varepsilon T_{+}^{0}\right)$-periodic solution is found by solving the equations

$$
\begin{aligned}
& x\left(\varepsilon T_{+}+\tau, \alpha, \varepsilon\right)=x\left(-T_{-}+\tau, \alpha, \varepsilon\right), \\
& T_{-}+\varepsilon T_{+}=T_{-}^{0}+\varepsilon T_{+}^{0} .
\end{aligned}
$$

Thus, the bifurcation equation is obtained by putting conditions $x\left(\varepsilon T_{+}+\tau, \alpha, \varepsilon\right)=x\left(-T_{-}+\right.$ $\tau, \alpha, \varepsilon), T_{-}+\varepsilon T_{+}=T_{-}^{0}+\varepsilon T_{+}^{0}$ and the fact that the points $x\left(\varepsilon T_{+}+\tau, \alpha, \varepsilon\right)$ and $x\left(-T_{-}+\tau, \alpha, \varepsilon\right)$ belong to $S$ together. Then, in Section 3, we use the Lyapunov-Schmidt method to prove that the above equations can be solved for $\left(T_{-}, T_{+}, \tau, \alpha\right) \simeq\left(T_{-}^{0}, T_{+}^{0}, \tau_{0}, 0\right)$ as functions of $\varepsilon>0$ small provided a certain Poincaré-Melnikov-like function has a simple zero. We will first study the case, that we call non-degenerate, when

$$
\frac{\partial}{\partial \alpha}\left[q_{+}\left(T_{+}(\alpha), \alpha\right)-q_{-}\left(-T_{-}(\alpha), \alpha\right)\right]_{\alpha=0} w \neq 0, \quad \forall w \in \mathbb{R}^{n-1} \text { such that } T_{-}^{\prime}(0) w=0 .
$$

Condition (1.4) has a simple geometrical meaning. The impact system (1.3) has a $T_{-}^{0}$ periodic solution if and only if the following condition holds:

$$
q_{+}\left(T_{+}(\alpha), \alpha\right)=q_{-}\left(-T_{-}(\alpha), \alpha\right), \quad T_{-}(\alpha)=T_{-}^{0} .
$$

Now, suppose there is a sequence $0 \neq \alpha_{n} \rightarrow 0$, as $n \rightarrow \infty$ such that (1.5) holds. Possibly passing to a subsequence we can suppose that $\lim _{n \rightarrow \infty} \frac{\alpha_{n}}{\left|\alpha_{n}\right|}=w,|w|=1$. Then, taking the limit in the equalities:

$$
\frac{q_{+}\left(T_{+}\left(\alpha_{n}\right), \alpha_{n}\right)-q_{-}\left(-T_{-}\left(\alpha_{n}\right), \alpha_{n}\right)}{\left|\alpha_{n}\right|}=0, \quad \frac{T_{-}\left(\alpha_{n}\right)-T_{-}^{0}}{\left|\alpha_{n}\right|}=0
$$

we see that condition (1.4) does not hold. Thus, (1.4) implies that, in a neighborhood of $\alpha=0$, there are no other $T_{-}^{0}$-periodic solutions of (1.3) apart from $q_{-}(t)$.

In Section 4, we define the adjoint system to the linearization of the impact system

$$
\begin{cases}\dot{x}=f_{-}(x), & \\ x(0)=q_{-}(0, \alpha), & x\left(-T_{-}(\alpha)\right)=R(x(0)), \\ G\left(x\left(-T_{-}(\alpha)\right)\right)=0, & -T_{-}(\alpha) \leq t \leq 0\end{cases}
$$

along the solution $x(t)=q_{-}(t, 0)$ and relate the Poincaré-Melnikov function obtained in Section 3 with the solutions of such an adjoint system. 
Section 5 is devoted to the extension of the result to the case (that we call degenerate) where $q_{+}\left(T_{+}(\alpha), \alpha\right)=q_{-}\left(-T_{-}(\alpha), \alpha\right)$ for any $\alpha \in I_{0}$. We will see that our results can be easily extended provided one of the following two conditions hold:

either $T_{-}^{\prime}(0) \neq 0$ or $T_{-}(\alpha)=T_{-}^{0}$ for any $\alpha \in I_{0}$.

Section 6 is devoted to the construction of some planar examples, although our results are given for an arbitrary finite dimension. Finally, the Appendix contains some technical proofs.

\section{The bifurcation equation}

We set $u_{+}(t, \alpha)=q_{+}\left(\varepsilon^{-1} t, \alpha\right), u_{-}(t, \alpha)=q_{-}(t, \alpha)$ and

$$
u(t, \alpha)= \begin{cases}u_{-}(t, \alpha) & \text { for }-T_{-}(\alpha) \leq t<0 \\ u_{+}(t, \alpha) & \text { for } 0 \leq t<\varepsilon T_{+}(\alpha) .\end{cases}
$$

Note that

$$
\left\{\begin{array}{l}
\varepsilon \dot{u}_{+}(t, \alpha)=f_{+}\left(u_{+}(t, \alpha)\right), \\
\dot{u}_{-}(t, \alpha)=f_{-}\left(u_{-}(t, \alpha)\right), \\
u_{+}(0, \alpha)=u_{-}(0, \alpha), \\
u_{+}\left(\varepsilon T_{+}(\alpha), \alpha\right), u_{-}\left(-T_{-}(\alpha), \alpha\right) \in S
\end{array}\right.
$$

and that $u(t, 0)$ is a continuous periodic solution, of period $T_{-}^{0}+\varepsilon T_{+}^{0}$, of the piecewise continuous singular system:

$$
\begin{cases}\varepsilon \dot{x}=f_{+}(x) & \text { for } x \in \Omega_{+}, \\ \dot{x}=f_{-}(x) & \text { for } x \in \Omega_{-} .\end{cases}
$$

Obviously, $u_{-}(t, \alpha)$ extends to a solution of the following impact system:

$$
\begin{cases}\dot{x}=f_{-}(x) & \text { for } x \in \Omega_{-}, \\ x\left(t^{+}\right)=q_{+}\left(T_{+}(\alpha), \alpha\right) & \text { when } x\left(t^{-}\right)=q_{-}(0, \alpha)\end{cases}
$$

that can be written as

$$
\begin{cases}\dot{x}=f_{-}(x) & \text { for } x \in \Omega_{-}, \\ x\left(t^{+}\right)=R\left(x\left(t^{-}\right)\right) & \text {when } x\left(t^{-}\right) \in U_{0} \cap S .\end{cases}
$$

Our purpose is to find a $T_{\varepsilon}$-periodic solution $x(t, \varepsilon)$ of system (1.1), which is orbitally close to $u(t, \alpha)$ for some $\alpha=\alpha(\varepsilon) \rightarrow 0$, as $\varepsilon \rightarrow 0^{+}$that is such that

$$
\sup _{-T_{-}^{0} \leq t \leq \varepsilon T_{+}^{0}}|x(t+\tau(\varepsilon), \varepsilon)-u(t, \alpha(\varepsilon))| \rightarrow 0 \quad \text { as } \varepsilon \rightarrow 0^{+}
$$

for some $(\tau(\varepsilon), \alpha(\varepsilon)) \rightarrow\left(\tau_{0}, 0\right)$ as $\varepsilon \rightarrow 0$. Thus, we may say that, in some sense, the impact periodic solution $u_{-}(t, 0)$ approximates the periodic solution $x(t, \varepsilon)$ of the singular perturbed equation (1.1). 
To this end, we first set $x(t+\tau)=x_{+}(t)+u_{+}(t, \alpha)$ in equation $\varepsilon \dot{x}=f_{+}(x)+\varepsilon g_{+}(t, x, \varepsilon)$. Then $x_{+}(t)$ satisfies

$$
\varepsilon \dot{x}-f_{+}^{\prime}\left(u_{+}(t, \alpha)\right) x=h_{+}(t, \tau, x, \alpha, \varepsilon)
$$

where

$$
\begin{aligned}
& h_{+}(t, \tau, x, \alpha, \varepsilon) \\
& \quad=f_{+}\left(x+u_{+}(t, \alpha)\right)-f_{+}\left(u_{+}(t, \alpha)\right)-f_{+}^{\prime}\left(u_{+}(t, \alpha)\right) x+\varepsilon g_{+}\left(t+\tau, x+u_{+}(t, \alpha), \varepsilon\right) .
\end{aligned}
$$

Since $u_{+}(0, \alpha)$ describes $U_{0} \cap S$, we consider (2.2) with the initial condition $x_{0}=0$. Let $X_{+}(t, \alpha)$ be the fundamental solution of $\dot{x}=f_{+}^{\prime}\left(q_{+}(t, \alpha)\right) x$, such that $X_{+}(0, \alpha)=\mathbb{I}$. Then $X_{+}\left(\varepsilon^{-1} t, \alpha\right)$ is the fundamental solution of $\varepsilon \dot{x}=f_{+}^{\prime}\left(u_{+}(t, \alpha)\right) x$, with $X_{+}(0, \alpha)=\mathbb{I}$. Let $T_{+}$be near $T_{+}^{0}$. By the variation of constants formula, the solution of (2.2) with the initial condition $x_{0}=0$ satisfies

$$
x_{+}(t)=\varepsilon^{-1} \int_{0}^{t} X_{+}\left(\varepsilon^{-1} t, \alpha\right) X_{+}^{-1}\left(\varepsilon^{-1} s, \alpha\right) h\left(s, \tau, x_{+}(s), \alpha, \varepsilon\right) d s .
$$

Thus, we conclude that for $\rho>0$ and $T_{+}$near $T_{+}^{0}$ equation $\varepsilon \dot{x}=f_{+}(x)+\varepsilon g(t, x, \varepsilon)$ has a solution $x(t)$ such that $\sup _{0 \leq t \leq \varepsilon T_{+}}\left|x(t+\tau)-u_{+}(t, \alpha)\right|<\rho$ if and only if the map $x(t) \mapsto \hat{x}(t)$ given by

$$
\hat{x}(t)=\varepsilon^{-1} \int_{0}^{t} X_{+}\left(\varepsilon^{-1} t, \alpha\right) X_{+}^{-1}\left(\varepsilon^{-1} s, \alpha\right) h(s, \tau, x(s), \alpha, \varepsilon) d s,
$$

has a fixed point whose sup-norm in $\left[0, \varepsilon T_{+}\right]$is smaller than $\rho$. To show that (2.3) has a fixed point of norm less than $\rho$, we set $y(t):=x\left(\varepsilon T_{+} t\right), t \in[0,1]$ and note that $x(t)$ is a fixed point of (2.3) of norm less than $\rho$, with $0 \leq t \leq \varepsilon T_{+}$, if and only if $y(t)$ is a fixed point of norm less than $\rho$ of the map:

$$
\hat{y}(t)=T_{+} \int_{0}^{t} X_{+}\left(T_{+} t, \alpha\right) X_{+}^{-1}\left(T_{+} \sigma, \alpha\right) h\left(\varepsilon T_{+} \sigma, \tau, y(\sigma), \alpha, \varepsilon\right) d \sigma
$$

$0 \leq t \leq 1$. Note that

$$
\begin{aligned}
h_{+}\left(\varepsilon T_{+} t, \tau, x, \alpha, \varepsilon\right)= & f_{+}\left(x+q_{+}\left(t T_{+}, \alpha\right)\right)-f_{+}\left(q_{+}\left(t T_{+}, \alpha\right)\right)-f_{+}^{\prime}\left(q_{+}\left(t T_{+}, \alpha\right)\right) x \\
& +\varepsilon g_{+}\left(\varepsilon t T_{+}+\tau, x+q_{+}\left(t T_{+}, \alpha\right), \varepsilon\right),
\end{aligned}
$$

and hence in the fixed-point equation (2.4), we may also take $\varepsilon \leq 0$. Then since $\left(x, T_{+}\right.$, $\alpha, \varepsilon) \mapsto h_{+}\left(\varepsilon T_{+} \tau, \tau, x, \alpha, \varepsilon\right), 0 \leq \tau \leq 1$ is a $C^{2}$-map and

$$
\left|h_{+}(t, \tau, x, \alpha, \varepsilon)\right| \leq \Delta(|x|)|x|+N_{g}|\varepsilon|,
$$

where

$$
\begin{aligned}
& N_{g}=\sup \left\{\left|g_{+}(t, \tilde{x}, \varepsilon)\right||t \in \mathbb{R},| \widetilde{x}\left|\leq \rho+\sup _{t \in\left[0, T_{+}(\alpha)\right], \alpha \in I_{0}}\right| q_{+}(t, \alpha)|,| \varepsilon \mid \leq \varepsilon_{0}\right\}, \\
& \Delta(\rho)=\sup \left\{\left|f^{\prime}\left(x+q_{+}(t, \alpha)\right)-f^{\prime}\left(q_{+}(t, \alpha)\right)\right|\left|t \in\left[0, T_{+}(\alpha)\right],\right| x \mid \leq \rho, \alpha \in I_{0}\right\},
\end{aligned}
$$


the map $y(t) \mapsto \hat{y}(t)$ is a $C^{2}$-contraction on the Banach space of bounded continuous functions on $[0,1]$ whose sup-norm is less than or equal to $\rho$ provided $\rho$ is sufficiently small, $T_{+}$is near $T_{+}^{0},|\varepsilon|$ is small, $\alpha \in I_{0}$ and $\tau \in \mathbb{R}$. Let $y_{+}\left(t, \tau, \alpha, T_{+}, \varepsilon\right)$ be the $C^{2}$-solution of the fixed point (2.4). We emphasize the fact that $\varepsilon$ may also be non-positive. Then $x_{+}(t, \tau, \alpha, \varepsilon):=y_{+}\left(\varepsilon^{-1} T_{+}^{-1} t, \tau, \alpha, T_{+}, \varepsilon\right)$ is a fixed point of (2.3) and

$$
x_{+}(\varepsilon t, \tau, \alpha, \varepsilon):=y_{+}\left(T_{+}^{-1} t, \tau, \alpha, T_{+}, \varepsilon\right)
$$

is $C^{2}$ in all parameters and $t$.

Writing $T_{+}^{-1} t$ in place of $t$ in (2.4) and using (2.5) we see that

$$
x_{+}(\varepsilon t, \tau, \alpha, \varepsilon)=\int_{0}^{t} X_{+}(t, \alpha) X_{+}^{-1}(s, \alpha) h_{+}\left(\varepsilon s, \tau, x_{+}(\varepsilon s, \tau, \alpha, \varepsilon), \alpha, \varepsilon\right) d s
$$

$0 \leq t \leq T_{+}$. We have, by definition, $x_{+}(0, \tau, \alpha, \varepsilon)+u_{+}(0, \alpha)=u_{+}(0, \alpha) \in S$ and

$$
x_{+}\left(\varepsilon T_{+}, \tau, \alpha, \varepsilon\right)+u_{+}\left(\varepsilon T_{+}, \alpha\right) \in S
$$

if and only if (recall that $\left.u_{+}\left(\varepsilon T_{+}, \alpha\right)=q_{+}\left(T_{+}, \alpha\right)\right)$

$$
G\left(q_{+}\left(T_{+}, \alpha\right)+\int_{0}^{T_{+}} X_{+}\left(T_{+}, \alpha\right) X_{+}^{-1}(s, \alpha) h_{+}\left(\varepsilon s, \tau, x_{+}(\varepsilon s, \tau, \alpha, \varepsilon), \alpha, \varepsilon\right) d s\right)=0 .
$$

We remark that equation (2.7) has meaning also when $\varepsilon<0$ but its relevance for our problem is only when $\varepsilon>0$.

As second step we consider the solution of the differential equation on $\Omega_{-}$:

$$
\dot{x}=f_{-}(x)+\varepsilon g_{-}(t, x, \varepsilon), \quad x(\tau)=q(0, \alpha)
$$

which is close to $u_{-}(t-\tau, \alpha)$ on $-T_{-}+\tau \leq t \leq \tau, T_{-} \simeq T_{-}^{0}$. Let $X_{-}(t, \alpha)$ be the fundamental solution of the linear system $\dot{x}=f_{-}^{\prime}\left(u_{-}(t, \alpha)\right) x$ such that $X_{-}(0, \alpha)=\mathbb{I}$. Setting $x(t+\tau)=x_{-}(t)+$ $u_{-}(t, \alpha)$ we see that (for $\left.t \in\left[-T_{-}, 0\right]\right) x_{-}(t)$ satisfies the equation:

$$
\left\{\begin{array}{l}
\dot{x}-f_{-}^{\prime}\left(u_{-}(t, \alpha)\right) x=h_{-}(t, \tau, x, \alpha, \varepsilon), \\
x(0)=0,
\end{array}\right.
$$

where

$$
\begin{aligned}
& h_{-}(t, \tau, x, \alpha, \varepsilon) \\
& \quad=f_{-}\left(x+u_{-}(t, \alpha)\right)-f_{-}\left(u_{-}(t, \alpha)\right)-f_{-}^{\prime}\left(u_{-}(t, \alpha)\right) x+\varepsilon g_{-}\left(t+\tau, x+u_{-}(t, \alpha), \varepsilon\right) .
\end{aligned}
$$

Again by the variation of constants formula we get the integral formula:

$$
x_{-}(t)=\int_{0}^{t} X_{-}(t, \alpha) X_{-}(s, \alpha)^{-1} h_{-}\left(s, \tau, x_{-}(s), \alpha, \varepsilon\right) d s
$$


which, as before, has a unique solution of norm less than a given, small, $\rho: x_{-}(t, \tau, \alpha, \varepsilon)$, with $-T_{-} \leq t \leq 0$. At $t=-T_{-}$the solution of (2.8) takes the value:

$$
-\int_{-T_{-}}^{0} X_{-}\left(-T_{-}, \alpha\right) X_{-}(s, \alpha)^{-1} h_{-}\left(s, \tau, x_{-}(s, \alpha, \varepsilon), \alpha, \varepsilon\right) d s .
$$

Now, we want to solve the equation

$$
x_{-}\left(-T_{-}, \tau, \alpha, \varepsilon\right)+u_{-}\left(-T_{-}, \alpha\right)=x_{+}\left(\varepsilon T_{+}, \tau, \alpha, \varepsilon\right)+u_{+}\left(\varepsilon T_{+}, \alpha\right)
$$

that is [again using $u_{+}\left(\varepsilon T_{+}, \alpha\right)=q_{+}\left(T_{+}, \alpha\right)$ and $u_{-}\left(-T_{-}, \alpha\right)=q_{-}\left(-T_{-}, \alpha\right)$ ]:

$$
\begin{aligned}
& q_{+}\left(T_{+}, \alpha\right)+\int_{0}^{T_{+}} X_{+}\left(T_{+}, \alpha\right) X_{+}^{-1}(s, \alpha) h_{+}\left(\varepsilon s, \tau, x_{+}(\varepsilon s, \tau, \alpha, \varepsilon), \alpha, \varepsilon\right) d s \\
& \quad=q_{-}\left(-T_{-}, \alpha\right)-\int_{-T_{-}}^{0} X_{-}\left(-T_{-}, \alpha\right) X_{-}(s, \alpha)^{-1} h_{-}\left(s, \tau, x_{-}(s, \tau, \alpha, \varepsilon), \alpha, \varepsilon\right) d s .
\end{aligned}
$$

Of course, when (2.9) holds, then (2.7) is equivalent to

$$
G\left(q_{-}\left(-T_{-}, \alpha\right)-\int_{-T_{-}}^{0} X_{-}\left(-T_{-}, \alpha\right) X_{-}(s, \alpha)^{-1} h_{-}\left(s, \tau, x_{-}(s, \tau, \alpha, \varepsilon), \alpha, \varepsilon\right) d s\right)=0
$$

So, our task reduces to solve the system formed by equations (2.9), (2.10) together with the period equation:

$$
T_{-}+\varepsilon T_{+}=T_{-}^{0}+\varepsilon T_{+}^{0}
$$

that is the equation $\mathcal{F}\left(T_{+}, T_{-}, \tau, \alpha, \varepsilon\right)=0$ where:

$$
\begin{aligned}
& \mathcal{F}\left(T_{+}, T_{-}, \tau, \alpha, \varepsilon\right) \\
& \quad:=\left(\begin{array}{c}
x_{-}\left(-T_{-}, \tau, \alpha, \varepsilon\right)+q_{-}\left(-T_{-}, \alpha\right)-x_{+}\left(\varepsilon T_{+}, \tau, \alpha, \varepsilon\right)-q_{+}\left(T_{+}, \alpha\right) \\
\left.G\left(q_{-}\left(-T_{-}, \alpha\right)-\int_{-T_{-}}^{0} X_{-}\left(-T_{-}, \alpha\right) X_{-}^{-1}(s, \alpha) h_{-}\left(s, \tau, x_{-}(s, \tau, \alpha, \varepsilon), \alpha, \varepsilon\right), \varepsilon\right) d s\right) \\
T_{-}-T_{-}^{0}+\varepsilon\left(T_{+}-T_{+}^{0}\right)
\end{array}\right) .
\end{aligned}
$$

According to the smoothness properties of $x_{-}(t, \tau, \alpha, \varepsilon)$ and $x_{+}(\varepsilon t, \tau, \alpha, \varepsilon)$, it results that $\mathcal{F}\left(T_{+}, T_{-}, \tau, \alpha, \varepsilon\right)$ is $C^{2}$.

\section{Solving $\mathcal{F}\left(T_{+}, T_{-}, \tau, \alpha, \varepsilon\right)=0$}

In this section, we will give a criterion to solve equation $\mathcal{F}\left(T_{+}, T_{-}, \tau, \alpha, \varepsilon\right)=0$ for $\left(T_{+}, T_{-}, \tau, \alpha\right)$ in terms of $\varepsilon$ for small $\varepsilon>0$. We will use a Crandall-Rabinowitz type result (see also [13, Theorem 4.1]) concerning the existence of a solution of a nonlinear equation having a manifold of fixed point at a certain value of a parameter.

Our result is as follows. Consider the linear system

$$
\left\{\begin{array}{l}
\psi^{*} \dot{q}_{+}\left(T_{+}^{0}, 0\right)=0 \\
\psi_{2}=\left[\psi^{*}+\psi_{1} G^{\prime}\left(q\left(-T_{-}^{0}, 0\right)\right)\right] \dot{q}_{-}\left(-T_{-}^{0}, 0\right) \\
\psi^{*}\left[\frac{\partial q_{-}}{\partial \alpha}\left(-T_{-}^{0}, 0\right)-\frac{\partial q_{+}}{\partial \alpha}\left(T_{+}^{0}, 0\right)\right]+\psi_{1} G^{\prime}\left(q\left(-T_{-}^{0}, 0\right)\right) \dot{q}_{-}\left(-T_{-}^{0}, 0\right) T_{-}^{\prime}(0)=0
\end{array}\right.
$$


We will prove that if (1.4) holds, system (3.1) has a unique solution, up to a multiplicative constant, and the following result holds:

Theorem 3.1 Assume condition (1.4) holds and let $\left(\psi, \psi_{1}, \psi_{2}\right) \in \mathbb{R}^{n} \times \mathbb{R} \times \mathbb{R}$ be the unique (up to a multiplicative constant) solution of (3.1). If the Poincaré-Melnikov function

$$
\begin{aligned}
\mathcal{M}(\tau):= & \psi * \int_{0}^{T_{+}^{0}} X_{+}\left(T_{+}^{0}, 0\right) X_{+}(s, 0)^{-1} g_{+}(\tau, u(0,0), 0) d s \\
& +\psi^{*} \int_{-T_{-}^{0}}^{0} X_{-}\left(-T_{-}^{0}, 0\right) X_{-}(s, 0)^{-1} g_{-}\left(s+\tau, u_{-}(s, 0), 0\right) d s \\
& +\psi_{1} G^{\prime}\left(q\left(-T_{-}^{0}, 0\right)\right) \int_{-}^{0} X_{-}^{0}\left(-T_{-}^{0}, 0\right) X_{-}^{-1}(s, 0) g_{-}\left(s+\tau, q_{-}(s, 0), 0\right) d s
\end{aligned}
$$

has a simple zero at $\tau=\tau_{0}$, then system (1.1) has a $T_{\varepsilon}$-periodic solution $x(t, \varepsilon)$ satisfying (2.1).

Proof To start with, we make few remarks on the functions $x_{ \pm}(t, \tau, \alpha, \varepsilon)$. First we note that when $\varepsilon=0$ equation $(2.8)$ reads

$$
\left\{\begin{array}{l}
\dot{x}=f_{-}\left(x+u_{-}(t, \alpha)\right)-f_{-}\left(u_{-}(t, \alpha)\right) \\
x(0)=0
\end{array}\right.
$$

which has the (unique) solution $x(t)=0$. Thus,

$$
x_{-}(t, \tau, \alpha, 0)=0 .
$$

Next, differentiating equation (2.8) with respect to $\varepsilon$ we see that $\frac{\partial x_{-}}{\partial \varepsilon}(t, \tau, \alpha, 0)$ satisfies the equation:

$$
\left\{\begin{array}{l}
\dot{x}-f_{-}^{\prime}\left(u_{-}(t, \alpha)\right) x=g_{-}\left(t+\tau, u_{-}(t, \alpha), 0\right) \\
x(0)=0 .
\end{array}\right.
$$

Hence,

$$
x_{-, \varepsilon}(t, \tau, \alpha, 0):=\frac{\partial x_{-}}{\partial \varepsilon}(t, \tau, \alpha, 0)=\int_{0}^{t} X_{-}(t, \alpha) X_{-}(s, \alpha)^{-1} g_{-}\left(s+\tau, u_{-}(s, \alpha), 0\right) d s
$$

Next, $x_{+}(0, \tau, \alpha, \varepsilon)=0$ by the definition and differentiating equation (2.6) with respect to $\varepsilon$ at $\varepsilon=0$ and using the equalities:

$$
x_{+}(0, \tau, \alpha, \varepsilon)=0, \quad h_{-, t}(0, \tau, 0, \alpha, 0)=0, \quad h_{-, x}(0, \tau, 0, \alpha, 0)=0
$$

we get

$$
t \dot{x}_{+}(0, \tau, \alpha, 0)=\int_{0}^{t} X_{+}(t, \alpha) X_{+}^{-1}(s, \alpha) g_{+}\left(\tau, u_{+}(0, \alpha), 0\right) d s
$$


So, equation (2.9) at $\varepsilon=0$ and $T_{ \pm}=T_{ \pm}(\alpha)$ becomes

$$
q_{-}\left(-T_{-}(\alpha), \alpha\right)=q_{+}\left(T_{+}(\alpha), \alpha\right)
$$

which is satisfied for $\alpha=0$. Now we look at equation (2.10). Since $h_{-}(t, \tau, 0, \alpha, 0)=0$, we see that when $\varepsilon=0$ and $T_{-}=T_{-}(\alpha)$ the equality is satisfied. As a consequence, we get

$$
\mathcal{F}\left(T_{+}(\alpha), T_{-}(\alpha), \tau, \alpha, 0\right)=\left(\begin{array}{c}
q_{-}\left(-T_{-}(\alpha), \alpha\right)-q_{+}\left(T_{+}(\alpha), \alpha\right) \\
0 \\
T_{-}(\alpha)-T_{-}^{0}
\end{array}\right)
$$

and $\mathcal{F}\left(T_{+}^{0}, T_{-}^{0}, \tau, 0,0\right)=0$. Next we look at derivatives of $\mathcal{F}$ with respect to $T_{+}, T_{-}, \alpha$ and $\varepsilon$ at the point $\left(T_{+}^{0}, T_{-}^{0}, \tau, 0,0\right)$. We have

$$
\begin{gathered}
\frac{\partial}{\partial T_{-}}\left[x_{-}\left(-T_{-}, \tau, \alpha, \varepsilon\right)+q_{-}\left(-T_{-}, \alpha\right)-x_{+}\left(\varepsilon T_{+}, \tau, \alpha, \varepsilon\right)-q_{+}\left(T_{+}, \alpha\right)\right] \\
\quad=-\dot{x}_{-}\left(-T_{-}, \tau, \alpha, \varepsilon\right)-\dot{q}_{-}\left(-T_{-}, \alpha\right) \rightarrow-\dot{q}_{-}\left(-T_{-}, \alpha\right), \quad \text { as } \varepsilon \rightarrow 0
\end{gathered}
$$

and similarly, using

$$
\begin{aligned}
\varepsilon \dot{x}_{+}\left(\varepsilon T_{+}, \tau, \alpha, \varepsilon\right)= & f\left(x_{+}\left(\varepsilon T_{+}, \tau, \alpha, \varepsilon\right)+q_{+}\left(T_{+}, \alpha\right)\right)-f\left(q_{+}\left(T_{+}, \alpha\right)\right) \\
& +\varepsilon g\left(t+\tau, x_{+}\left(\varepsilon T_{+}, \tau, \alpha, \varepsilon\right)+q_{+}\left(T_{+}, \alpha\right), \varepsilon\right),
\end{aligned}
$$

we get

$$
\begin{gathered}
\frac{\partial}{\partial T_{+}}\left[x_{-}\left(-T_{-}, \tau, \alpha, \varepsilon\right)+q_{-}\left(-T_{-}, \alpha\right)-x_{+}\left(\varepsilon T_{+}, \tau, \alpha, \varepsilon\right)-q_{+}\left(T_{+}, \alpha\right)\right] \\
\quad=-\varepsilon \dot{x}_{+}\left(\varepsilon T_{+}, \tau, \alpha, \varepsilon\right)-\dot{q}_{+}\left(T_{+}, \alpha\right) \rightarrow-\dot{q}_{+}\left(T_{+}, \alpha\right), \quad \text { as } \varepsilon \rightarrow 0 .
\end{gathered}
$$

Next

$$
\begin{aligned}
\frac{\partial}{\partial \alpha} & {\left[x_{-}\left(-T_{-}, \tau, \alpha, \varepsilon\right)+q_{-}\left(-T_{-}, \alpha\right)-x_{+}\left(\varepsilon T_{+}, \tau, \alpha, \varepsilon\right)-q_{+}\left(T_{+}, \alpha\right)\right] } \\
& \rightarrow \frac{\partial q_{-}}{\partial \alpha}\left(-T_{-}, \alpha\right)-\frac{\partial q_{+}}{\partial \alpha}\left(T_{+}, \alpha\right), \quad \text { as } \varepsilon \rightarrow 0,
\end{aligned}
$$

and

$$
\frac{\partial}{\partial \tau}\left[x_{-}\left(-T_{-}, \tau, \alpha, \varepsilon\right)+q_{-}\left(-T_{-}, \alpha\right)-x_{+}\left(\varepsilon T_{+}, \tau, \alpha, \varepsilon\right)-q_{+}\left(T_{+}, \alpha\right)\right] \rightarrow 0 \quad \text { as } \varepsilon \rightarrow 0 .
$$

So, the Jacobian matrix $L$ of $\mathcal{F}$ at the point $\left(T_{+}^{0}, T_{-}^{0}, \tau, 0,0\right)$ is

$$
\begin{aligned}
L & :=\frac{\partial \mathcal{F}}{\partial\left(T_{+}, T_{-}, \tau, \alpha\right)}\left(T_{+}^{0}, T_{-}^{0}, \tau, 0,0\right) \\
& =\left(\begin{array}{cccc}
-\dot{q}_{+}\left(T_{+}^{0}, 0\right) & -\dot{q}_{-}\left(-T_{-}^{0}, 0\right) & 0 & \frac{\partial q_{-}}{\partial \alpha}\left(-T_{-}^{0}, 0\right)-\frac{\partial q_{+}}{\partial \alpha}\left(T_{+}^{0}, 0\right) \\
0 & -G^{\prime}\left(q\left(-T_{-}^{0}, 0\right)\right) \dot{q}_{-}\left(-T_{-}^{0}, 0\right) & 0 & G^{\prime}\left(q\left(-T_{-}^{0}, 0\right)\right) \frac{\partial q_{-}}{\partial \alpha}\left(-T_{-}^{0}, 0\right) \\
0 & 1 & 0 & 0
\end{array}\right)
\end{aligned}
$$


and $\left(\mu_{+}, \mu_{-}, \tau, w\right) \in \mathbb{R} \times \mathbb{R} \times \mathbb{R} \times \mathbb{R}^{n-1}$ belongs to the kernel $\mathcal{N} L$ of $L$ if and only if

$$
\left\{\begin{array}{l}
\mu_{-}=0 \\
{\left[\frac{\partial q_{-}}{\partial \alpha}\left(-T_{-}^{0}, 0\right)-\frac{\partial q_{+}}{\partial \alpha}\left(T_{+}^{0}, 0\right)\right] w=\dot{q}_{+}\left(T_{+}^{0}, 0\right) \mu_{+}} \\
G^{\prime}\left(q_{-}\left(-T_{-}^{0}, 0\right)\right) \frac{\partial q_{-}}{\partial \alpha}\left(-T_{-}^{0}, 0\right) w=0 .
\end{array}\right.
$$

From $G\left(q_{-}\left(-T_{-}(\alpha), \alpha\right)\right)=0$, we get

$$
G^{\prime}\left(q\left(-T_{-}^{0}, 0\right)\right)\left[-\dot{q}_{-}\left(-T_{-}^{0}, 0\right) T_{-}^{\prime}(0)+\frac{\partial q_{-}}{\partial \alpha}\left(-T_{-}^{0}, 0\right)\right]=0
$$

thus, on account of the transversality condition $G^{\prime}\left(q\left(T_{-}^{0}, 0\right)\right) \dot{q}_{-}\left(-T_{-}^{0}, 0\right) \neq 0,(3.4)$ is equivalent to

$$
\left\{\begin{array}{l}
{\left[\frac{\partial q_{-}}{\partial \alpha}\left(-T_{-}^{0}, 0\right)-\frac{\partial q_{+}}{\partial \alpha}\left(T_{+}^{0}, 0\right)\right] w=\dot{q}_{+}\left(T_{+}^{0}, 0\right) \mu_{+}} \\
T_{-}^{\prime}(0) w=0 \\
\mu_{-}=0
\end{array}\right.
$$

Next, from $G\left(q_{+}\left(T_{+}(\alpha), \alpha\right)\right)=0$, we get

$$
G^{\prime}\left(q\left(T_{+}^{0}, 0\right)\right)\left[\dot{q}_{+}\left(T_{+}^{0}, 0\right) T_{+}^{\prime}(0)+\frac{\partial q_{+}}{\partial \alpha}\left(T_{+}^{0}, 0\right)\right]=0
$$

then subtracting (3.5) from (3.7) and using $q\left(T_{+}^{0}, 0\right)=q\left(-T_{-}^{0}, 0\right)$ we obtain:

$$
\begin{gathered}
G^{\prime}\left(q\left(T_{+}^{0}, 0\right)\right)\left[\dot{q}_{+}\left(T_{+}^{0}, 0\right) T_{+}^{\prime}(0)+\dot{q}_{-}\left(-T_{-}^{0}, 0\right) T_{-}^{\prime}(0)\right] \\
\quad=G^{\prime}\left(q\left(T_{+}^{0}, 0\right)\right)\left[\frac{\partial q_{-}}{\partial \alpha}\left(-T_{-}^{0}, 0\right)-\frac{\partial q_{+}}{\partial \alpha}\left(T_{+}^{0}, 0\right)\right] .
\end{gathered}
$$

So, if $w \in \mathbb{R}^{n-1}$ satisfies (3.6), we see that

$$
G^{\prime}\left(q\left(T_{+}^{0}, 0\right)\right) \dot{q}_{+}\left(T_{+}^{0}, 0\right) T_{+}^{\prime}(0) w=G^{\prime}\left(q\left(T_{+}^{0}, 0\right)\right) \dot{q}_{+}\left(T_{+}^{0}, 0\right) \mu_{+}
$$

and then, on account of transversality, $T_{+}^{\prime}(0) w=\mu_{+}$. Summarizing, we have seen that, if $\left(\mu_{+}, \mu_{-}, \tau, w\right) \in \mathcal{N} L$ then $\mu_{+}=T_{+}^{\prime}(0) w, \mu_{-}=0$ and $w \in \mathbb{R}^{n-1}$ satisfies

$$
\left\{\begin{array}{l}
{\left[\frac{\partial q_{-}}{\partial \alpha}\left(-T_{-}^{0}, 0\right)-\frac{\partial q_{+}}{\partial \alpha}\left(T_{+}^{0}, 0\right)\right] w=\dot{q}_{+}\left(T_{+}^{0}, 0\right) T_{+}^{\prime}(0) w} \\
T_{-}^{\prime}(0) w=0 .
\end{array}\right.
$$

On the other hand, if $w \in \mathbb{R}^{n-1}$ satisfies (3.8), then $\left(T_{+}^{\prime}(0) w, 0, \tau, w\right)$ belongs to $\mathcal{N} L$. So $\mathcal{N} L=\operatorname{span}\{(0,0,1,0)\}$ if and only if system (3.8) has the trivial solution $w=0$ only. But (3.8) is equivalent to

$$
\left\{\begin{array}{l}
\frac{\partial}{\partial \alpha}\left[q_{-}\left(-T_{-}(\alpha), \alpha\right)-q_{+}\left(T_{+}(\alpha), \alpha\right)\right]_{\alpha=0} w=0, \\
T_{-}^{\prime}(0) w=0,
\end{array}\right.
$$

and hence (3.8) has the trivial solution if and only if the non-degenerateness condition (1.4) holds. We emphasize the fact that, assuming condition (1.4), equation $\mathcal{F}\left(T_{+}, T_{-}, \tau, \alpha, 0\right)=$ 
0 has the manifold of fixed points $\left(T_{+}, T_{-}, \tau, \alpha\right)=\left(T_{+}^{0}, T_{-}^{0}, \tau, 0\right)$ and the linearization of $\mathcal{F}$ at these points is Fredholm with index zero with the one-dimensional kernel $\operatorname{span}\{(0,0,1,0)\}$. Hence, there is a unique vector, up to a multiplicative constant, $\widetilde{\psi} \in \mathbb{R}^{n+2}$ such that $\widetilde{\psi}^{*} L=0$, i.e.,

$$
\widetilde{\psi}^{*}\left(\begin{array}{cccc}
-\dot{q}_{+}\left(T_{+}^{0}, 0\right) & -\dot{q}_{-}\left(-T_{-}^{0}, 0\right) & 0 & \frac{\partial q_{-}}{\partial \alpha}\left(-T_{-}^{0}, 0\right)-\frac{\partial q_{+}}{\partial \alpha}\left(T_{+}^{0}, 0\right) \\
0 & -G^{\prime}\left(q\left(-T_{-}^{0}, 0\right)\right) \dot{q}_{-}\left(-T_{-}^{0}, 0\right) & 0 & G^{\prime}\left(q\left(-T_{-}^{0}, 0\right)\right) \frac{\partial q_{-}}{\partial \alpha}\left(-T_{-}^{0}, 0\right) \\
0 & 1 & 0 & 0
\end{array}\right)=0 .
$$

Writing $\widetilde{\psi}^{*}=\left(\psi^{*}, \psi_{1}, \psi_{2}\right), \psi \in \mathbb{R}^{n}, \psi_{1}, \psi_{2} \in \mathbb{R}$ we see that $\psi, \psi_{1}, \psi_{2}$ satisfy (3.1). This proves the claim before the statement of Theorem 3.1.

We recall that our purpose is to solve the equation $\mathcal{F}\left(T_{+}, T_{-}, \tau, \alpha, \varepsilon\right)=0$ for $\varepsilon \neq 0$ and that $\mathcal{F}\left(T_{+}, T_{-}, \tau, \alpha, 0\right)=0$ has the one-dimensional manifold of solutions $\left(T_{+}, T_{-}, \tau, \alpha\right)=$ $\left(T_{+}^{0}, T_{-}^{0}, \tau, 0\right)$ and its linearization along the points of this manifold is Fredholm with the one-dimensional kernel span $\{(0,0,1,0)\}$. Hence, we are in position of applying the following result that has been more or less proved in [13].

Theorem 3.2 Let, $X, Y$ be Banach spaces and $F: X \times \mathbb{R} \rightarrow Y$ a $C^{2}$-map such that $F(x, 0)=$ 0 has a $C^{2}$,d-dimensional, manifold of solutions $\mathcal{M}=\left\{x=\xi(\mu) \mid \mu \in \mathbb{R}^{d}\right\}$. Assume that for any $\mu$ in a neighborhood of $\mu=0$ the linearization $L(\mu)=D_{1} F(\xi(\mu), 0)$ has the null space $T_{\xi(\mu)} \mathcal{M}=\operatorname{span}\left\{\xi^{\prime}(\mu)\right\}$. Assume further that $L(\mu)$ is Fredholm with index zero and let $\Pi(\mu): Y \rightarrow \mathcal{R} L(\mu)$ a projection of $Y$ onto the range of $L(\mu)$. Then if the Poincaré-Melnikov function

$$
[\mathbb{I}-\Pi(\mu)] D_{2} F(\xi(\mu), 0)
$$

has a simple zero at $\mu=0$, there exists $\bar{\varepsilon}>0$ and a unique map $(-\bar{\varepsilon}, \bar{\varepsilon}) \mapsto x(\varepsilon) \in X$ such that $F(x(\varepsilon), \varepsilon)=0$. Moreover, $D_{1} F(x(\varepsilon), \varepsilon)$ is an isomorphism for $\varepsilon \neq 0$.

Actually the statement in [13, Theorem 4.1] is slightly different from the above. Hence, we give a proof of Theorem 3.2 in the Appendix.

We apply Theorem 3.2 to the map $\mathcal{F}\left(T_{+}, T_{-}, \tau, \alpha, \varepsilon\right)$ with $\mu=\tau$. Then $L(\tau)=L$ is independent of $\tau$, and hence so is $\Pi(\tau)=\Pi$. Next $[\mathbb{I}-\Pi] z=\frac{\widetilde{\psi}^{*} z}{|\widetilde{\psi}|^{2}} \widetilde{\psi}$ where $\mathcal{R} L=\{\widetilde{\psi}\}^{\perp}$ and $\widetilde{\psi}^{*}=\left(\psi^{*}, \psi_{1}, \psi_{2}\right) \in \mathbb{R}^{n+2}, \psi \in \mathbb{R}^{n}, \psi_{1}, \psi_{2} \in \mathbb{R}$, is any vector satisfying (3.1). To apply Theorem 3.2, we look at the derivative of $\mathcal{F}\left(T_{+}^{0}, T_{-}^{0}, \tau, 0, \varepsilon\right)$ with respect to $\varepsilon$ at $\varepsilon=0$. First, we have:

$$
\begin{aligned}
& \left.\frac{\partial\left[x_{+}\left(\varepsilon T_{+}, \alpha, \varepsilon\right)-x_{-}\left(-T_{-}, \alpha, \varepsilon\right)\right]}{\partial \varepsilon}\right|_{\varepsilon=0} \\
& =\int_{0}^{T_{+}} X_{+}\left(T_{+}, \alpha\right) X_{+}(s, \alpha)^{-1} g_{+}(\tau, u(0, \alpha), 0) d s \\
& \quad+\int_{-T_{-}}^{0} X_{-}\left(-T_{-}, \alpha\right) X_{-}(s, \alpha)^{-1} g_{-}\left(s+\tau, u_{-}(s, \alpha), 0\right) d s
\end{aligned}
$$

whereas differentiating (2.10) with respect to $\varepsilon$ at $\varepsilon=0$ we get

$$
{ }_{-} G^{\prime}\left(q_{-}\left(-T_{-}, \alpha\right)\right) \int_{-T_{-}}^{0} X_{-}\left(-T_{-}, \alpha\right) X_{-}^{-1}(s, \alpha) g_{-}\left(s+\tau, q_{-}(s, \alpha), 0\right) d s .
$$


We obtain then

$$
\begin{aligned}
& \frac{\partial \mathcal{F}}{\partial \varepsilon}\left(T_{+}^{0}, T_{-}^{0}, \tau, 0,0\right) \\
& \quad=\left(\begin{array}{c}
-\int_{0}^{T_{+}^{0}} X_{+}\left(T_{+}^{0}, 0\right) X_{+}(s, 0)^{-1} g_{+}\left(\tau, q_{+}(0,0), 0\right) d s \\
-\int_{-T_{-}^{0}}^{0} X_{-}\left(-T_{-}^{0}, 0\right) X_{-}(s, 0)^{-1} g_{-}\left(s+\tau, q_{-}(s, 0), 0\right) d s \\
-G^{\prime}\left(q\left(-T_{-}^{0}, 0\right)\right) \int_{-T_{-}^{0}}^{0} X_{-}\left(-T_{-}^{0}, 0\right) X_{-}^{-1}(s, 0) g_{-}\left(s+\tau, q_{-}(s, 0), 0\right) d s \\
0
\end{array}\right)
\end{aligned}
$$

and then the Poincaré-Melnikov function is:

$$
\begin{aligned}
\mathcal{M}(\tau) & \\
:= & \psi^{*} \int_{0}^{T_{+}^{0}} X_{+}\left(T_{+}^{0}, 0\right) X_{+}(s, 0)^{-1} g_{+}(\tau, u(0,0), 0) d s \\
& +\psi^{*} \int_{-T_{-}^{0}}^{0} X_{-}\left(-T_{-}^{0}, 0\right) X_{-}(s, 0)^{-1} g_{-}\left(s+\tau, u_{-}(s, 0), 0\right) d s \\
& +\psi_{1} G^{\prime}\left(q\left(-T_{-}^{0}, 0\right)\right) \int_{-T_{-}^{0}}^{0} X_{-}\left(-T_{-}^{0}, 0\right) X_{-}^{-1}(s, 0) g_{-}\left(s+\tau, q_{-}(s, 0), 0\right) d s .
\end{aligned}
$$

The conclusion of Theorem 3.1 now easily follows from (3.9) and Theorem 3.2.

\section{Poincaré-Melnikov function and adjoint system}

In this section, we want to give a suitable definition of the adjoint system of the linearization of (1.6) along $q_{-}(t)$ in such a way that the Poincaré-Melnikov function (3.2) can be put in relation with the solutions of such an adjoint system.

Let $R: U_{0} \cap S \rightarrow U_{+} \cap S$ be the $C^{1}$-map defined in Introduction and recall the impact equation (1.6):

$$
\left\{\begin{array}{l}
\dot{x}=f_{-}(x), \\
x(0)=q_{-}(0, \alpha) \in S \cap U_{0}, \\
x(-T(\alpha))=R(x(0)), \\
G(x(-T(\alpha)))=0 \\
-T(\alpha) \leq t \leq 0 .
\end{array}\right.
$$

For $\alpha=0$, (4.1) has the solution $x(t)=q_{-}(t, 0),-T_{-}^{0} \leq t \leq 0$. We let $x(t, \alpha)$ denote the solution of the impact system (4.1) on [ $-T(\alpha), 0]$. Then its derivative with respect to $\alpha$ at $\alpha=0$ satisfies the linearized equation:

$$
\left\{\begin{array}{l}
\dot{u}=f_{-}^{\prime}\left(q_{-}(t, 0)\right) u \\
u(0)=\frac{\partial q_{-}}{\partial \alpha}(0,0) \\
R^{\prime}(q(0,0)) u(0)=u\left(-T_{-}^{0}\right)-\dot{q}_{-}\left(-T_{-}^{0}, 0\right) T_{1}, \\
G^{\prime}\left(q_{-}\left(-T_{-}^{0}, 0\right)\right)\left[u\left(-T_{-}^{0}\right)-\dot{q}_{-}\left(-T_{-}^{0}, 0\right) T_{1}\right]=0, \\
T^{\prime}(0)=T_{1}: \mathbb{R}^{n-1} \rightarrow \mathbb{R} .
\end{array}\right.
$$


Next, recalling (1.1), we consider a perturbed impact system of (4.1) (see also (2.8)) of the form

$$
\left\{\begin{array}{l}
\dot{x}=f_{-}(x)+\varepsilon g_{-}(t+\tau, x, \varepsilon) \\
x(0)=q_{-}(0, \alpha) \in S \cap U_{0} \\
x(-T(\alpha, \varepsilon))=R(\tau ; x(0), \varepsilon) \\
G(x(-T(\alpha, \varepsilon)))=0 \\
-T(\alpha, \varepsilon) \leq t \leq 0
\end{array}\right.
$$

where $R: \mathbb{R} \times U_{0} \cap S \times(-\delta, \delta) \rightarrow U_{+} \cap S$ is defined as follows: $R(\tau ; \xi, \varepsilon)=x_{+}\left(\varepsilon T_{+}(\xi, \tau, \varepsilon), \tau, \varepsilon\right)$ and $x_{+}(t, \tau, \varepsilon)$ is the solution of

$$
\begin{aligned}
& \varepsilon \dot{x}=f_{+}(x)+\varepsilon g_{+}(t+\tau, x, \varepsilon), \\
& x(0)=\xi .
\end{aligned}
$$

Note that $R$ is a $C^{2}$-map on $\mathbb{R} \times U_{0} \cap S \times \mathbb{R}$ taking values on $U_{+} \cap S$ and $R(\tau ; q(0, \alpha), 0)=$ $q_{+}\left(T_{+}(\alpha), \alpha\right)$; moreover, when $g_{+}$is autonomous then $R$ is independent of $\tau$, so we may take $\tau=0$ in its definition. We recall that for simplicity we write $R(\xi)$ instead of $R(\tau ; \xi, 0), \xi \in S$. To study the problem of existence of solutions of system (4.3), we are then led to find conditions on $h(t), d$ and $T_{1}$ so that the non-homogeneous linear equation:

$$
\left\{\begin{array}{l}
\dot{u}-f_{-}^{\prime}\left(q_{-}(t, 0)\right) u=h(t), \\
u(0)=\frac{\partial q_{-}}{\partial \alpha}(0,0) \theta, \quad \theta \in \mathbb{R}^{n-1}, \\
u\left(-T_{-}^{0}\right)-\dot{q}_{-}\left(-T_{-}^{0}, 0\right) T-R^{\prime}(q(0,0)) u(0)=d \in \mathbb{R}^{n}, \\
G^{\prime}\left(q_{-}\left(-T_{-}^{0}, 0\right)\right)\left[u\left(-T_{-}^{0}\right)-\dot{q}_{-}\left(-T_{-}^{0}, 0\right) T\right]=0, \\
T=T_{1}
\end{array}\right.
$$

has a solution $(u(t), \theta, T)$. Let us comment on equation (4.4) (and similarly on (4.2)) that condition $u\left(-T_{-}^{0}\right)-\dot{q}_{-}\left(-T_{-}^{0}, 0\right) T-R^{\prime}(q(0,0)) u(0)=d$ only involves the derivative of $R(\xi)$ on the tangent space $T_{\xi} S$ since $u(0)=\frac{\partial q_{-}}{\partial \alpha}(0,0) \in T_{\xi} S, \xi=q_{-}(0,0)$. So, it is independent of any extension we take of $R(\xi)$ to a neighborhood of $q_{-}(0,0)$. We also note that for simplicity we denote again by $T_{1}$ the value of the linear functional $T_{1}$ in (4.4).

Since $G\left(R\left(q_{-}(0, \alpha)\right)\right)=0$, we get

$$
G^{\prime}\left(R\left(q_{-}(0,0)\right)\right) R^{\prime}\left(q_{-}(0,0)\right) \frac{\partial q_{-}}{\partial \alpha}(0,0) \theta=0
$$

for any $\theta \in \mathbb{R}^{n-1}$ and then

$$
\begin{aligned}
G^{\prime}\left(R\left(q_{-}(0,0)\right)\right) d & =G^{\prime}\left(R\left(q_{-}(0,0)\right)\right)\left[u\left(-T_{-}^{0}\right)-\dot{q}_{-}\left(-T_{-}^{0}, 0\right) T-R^{\prime}(q(0,0)) \frac{\partial q_{-}}{\partial \alpha}(0,0) \theta\right] \\
& =0 .
\end{aligned}
$$

So, if equation (4.4) has a solution, we must necessarily have

$$
G^{\prime}\left(R\left(q_{-}(0,0)\right)\right) d=0 \quad\left[\Leftrightarrow \quad G^{\prime}\left(q_{+}\left(T_{+}^{0}, 0\right)\right) d=0\right] .
$$


Next, we define two Hilbert spaces:

$$
\begin{aligned}
& X:=\left\{(u, \theta, T) \in W^{1,2}\left(\left[-T_{-}^{0}, 0\right], \mathbb{R}^{n}\right) \times \mathbb{R}^{n-1} \times \mathbb{R} \mid u(0)=\frac{\partial q_{-}}{\partial \alpha}(0,0) \theta\right\} \\
& Y:=\left\{(h, d, T) \in L^{2}\left(\left[-T_{-}^{0}, 0\right], \mathbb{R}^{n}\right) \times \mathbb{R}^{n-1} \times \mathbb{R} \times \mathbb{R} \mid G^{\prime}\left(R\left(q_{-}(0,0)\right)\right) d=0\right\} .
\end{aligned}
$$

Note $Y$ is a Hilbert space and $X$ is a closed subspace of a Hilbert space $W^{1,2}\left(\left[-T_{-}^{0}, 0\right], \mathbb{R}^{n}\right) \times$ $\mathbb{R}^{n-1} \times \mathbb{R}$. Then (4.4) can be written as

$$
A(u, \theta, T)=\left(h, d, 0, T_{1}\right)
$$

with

$$
A(u, \theta, T):=\left(\begin{array}{c}
\dot{u}-f_{-}^{\prime}\left(q_{-}(t, 0)\right) u \\
u\left(-T_{-}^{0}\right)-\dot{q}_{-}\left(-T_{-}^{0}, 0\right) T-R^{\prime}(q(0,0)) u(0) \\
G^{\prime}\left(q_{-}\left(-T_{-}^{0}, 0\right)\right)\left[u\left(-T_{-}^{0}\right)-\dot{q}_{-}\left(-T_{-}^{0}, 0\right) T\right] \\
T
\end{array}\right)
$$

and $A: X \rightarrow Y$.

Lemma 4.1 The range $\mathcal{R} A$ is closed.

Proof Indeed, let $A\left(u_{n}, \theta_{n}, T_{n}\right)=\left(h_{n}, d_{n}, 0, T_{1}^{n}\right) \rightarrow\left(\bar{h}, \bar{d}, 0, \bar{T}_{1}\right)$. Then

$$
u_{n}(t)=\frac{\partial q_{-}}{\partial \alpha}(t, 0) \theta_{n}-\int_{t}^{0} X_{-}(t) X_{-}^{-1}(t, s) h_{n}(s) d s
$$

and

$$
\left\{\begin{array}{l}
R^{\prime}(q(0,0)) \frac{\partial q_{-}}{\partial \alpha}(0,0) \theta_{n}-\frac{\partial q_{-}}{\partial \alpha}\left(-T_{-}^{0}, 0\right) \theta_{n} \\
\quad=-d_{n}-\int_{-T_{-}^{0}}^{0} X_{-}\left(-T_{-}^{0}, 0\right) X_{-}(s, 0)^{-1} h_{n}(s) d s-\dot{q}_{-}\left(-T_{-}^{0}, 0\right) T_{1}^{n}, \\
G^{\prime}\left(q_{-}\left(-T_{-}^{0}, 0\right)\right) d_{n}=0 .
\end{array}\right.
$$

Since

$$
\begin{gathered}
-d_{n}-\int_{-T_{-}^{0}}^{0} X_{-}\left(-T_{-}^{0}, 0\right) X_{-}(s, 0)^{-1} h_{n}(s) d s-\dot{q}_{-}\left(-T_{-}^{0}, 0\right) T_{1}^{n} \\
\rightarrow-\bar{d}-\int_{-T_{-}^{0}}^{0} X_{-}\left(-T_{-}^{0}, 0\right) X_{-}(s, 0)^{-1} \bar{h}(s) d s-\dot{q}_{-}\left(-T_{-}^{0}, 0\right) \bar{T}_{1},
\end{gathered}
$$

and $\mathcal{R}\left[R^{\prime}(q(0,0)) \frac{\partial q_{-}}{\partial \alpha}(0,0) \cdot-\frac{\partial q_{-}}{\partial \alpha}\left(-T_{-}^{0}, 0\right) \cdot\right]$ is closed, then $G^{\prime}\left(q_{-}\left(-T_{-}^{0}, 0\right)\right) \bar{d}=0$ and there exists $\bar{\theta} \in \mathbb{R}^{n-1}$ so that

$$
\begin{aligned}
& R^{\prime}(q(0,0)) \frac{\partial q_{-}}{\partial \alpha}(0,0) \bar{\theta}-\frac{\partial q_{-}}{\partial \alpha}\left(-T_{-}^{0}, 0\right) \bar{\theta} \\
& \quad=-\bar{d}-\int_{-T_{-}^{0}}^{0} X_{-}\left(-T_{-}^{0}, 0\right) X_{-}(s, 0)^{-1} \bar{h}(s) d s-\dot{q}_{-}\left(-T_{-}^{0}, 0\right) \bar{T}_{1} .
\end{aligned}
$$


By taking

$$
\bar{u}(t):=\frac{\partial q_{-}}{\partial \alpha}(t, 0) \bar{\theta}-\int_{t}^{0} X_{-}(t) X_{-}^{-1}(t, s) \bar{h}(s) d s, \quad \bar{T}=\bar{T}_{1}
$$

we derive $\left(\bar{h}, \bar{d}, 0, \bar{T}_{1}\right)=A(\bar{u}, \bar{\theta}, \bar{T}) \in \mathcal{R} A$. The proof is finished.

Next, we prove the following result.

Proposition 4.1 Let $(h, d, T) \in Y$. Then the inhomogeneous system (4.4) has a solution $(u(t), \theta, T) \in X$ if and only if equation

$$
\int_{-T_{-}^{0}}^{0} v(t)^{*} h(t) d t+\psi^{*} d+\psi_{2} T_{1}=0
$$

holds for any solution $v(t)$ of the adjoint system

$$
\left\{\begin{array}{l}
\dot{v}(t)+f_{-}^{\prime}\left(q_{-}(t, 0)\right)^{*} v(t)=0, \\
{\left[\frac{\partial q_{-}}{\partial \alpha}(0,0)\right]^{*}\left[v(0)-R^{\prime}\left(q_{-}(0,0)\right)^{*} \psi\right]=0,} \\
v\left(-T_{-}^{0}\right)=\psi+\psi_{1} G^{\prime}\left(q_{-}\left(-T_{-}^{0}, 0\right)\right)^{*}, \\
\psi^{*} \dot{q}_{+}\left(T_{+}^{0}, 0\right)=0
\end{array}\right.
$$

and $\psi_{2}=\psi^{*} \dot{q}_{-}\left(-T_{-}^{0}, 0\right)+\psi_{1} G^{\prime}\left(q_{-}\left(-T_{-}^{0}, 0\right)\right) \dot{q}_{-}\left(-T_{-}^{0}, 0\right)$.

Proof Before starting with the proof we observe that, because of $G^{\prime}\left(q_{+}\left(T_{+}^{0}, 0\right)\right) d=0, \psi$ is not uniquely determined by equation (4.5) since changing it with $\psi+\lambda G^{\prime}\left(q_{-}\left(-T_{-}^{0}, 0\right)\right)^{*}$, $\lambda \in \mathbb{R}$, the equation remains the same. So, in equation (4.5), we look for $\psi$ in a subspace of $\mathbb{R}^{n}$ which is transverse to $G^{\prime}\left(q_{-}\left(-T_{-}^{0}, 0\right)\right)^{*}$. It turns out that the best choice, from a computational point of view, is to take $\psi$ so that $\psi^{*} \dot{q}_{+}\left(T_{+}^{0}, 0\right)=0$ (see equation (3.1)).

First, we prove necessity. Assume that (4.4) can be solved for $(u, \theta, T) \in X$ and let $\left(v(t), \psi, \psi_{1}\right), v \in W^{1,2}\left(\left[-T_{-}^{0}, 0\right], \mathbb{R}^{n}\right)$, be a solution of equation (4.6). Then

$$
\begin{aligned}
& h(t)=\dot{u}(t)-f^{\prime}\left(q_{-}(t, 0)\right) u(t), \\
& d=u\left(-T_{-}^{0}\right)-\dot{q}_{-}\left(-T_{-}^{0}, 0\right) T-R^{\prime}(q(0,0)) \frac{\partial q_{-}}{\partial \alpha}(0,0) \theta, \\
& 0=G^{\prime}\left(q_{-}\left(-T_{-}^{0}, 0\right)\right)\left[u\left(-T_{-}^{0}\right)-\dot{q}_{-}\left(-T_{-}^{0}, 0\right) T\right], \\
& T_{1}=T .
\end{aligned}
$$

Plugging these equalities in the left-hand side of (4.5) and integrating by parts, (4.5) reads

$$
\begin{aligned}
& v(0)^{*} \frac{\partial q_{-}}{\partial \alpha}(0,0) \theta-v\left(-T_{-}^{0}\right)^{*} u\left(-T_{-}^{0}\right)-\int_{-T_{-}^{0}}^{0}\left[\dot{v}(t)+f_{-}^{\prime}\left(q_{-}(t, 0)\right)^{*} v(t)\right]^{*} u(t) d t \\
& +\psi^{*}\left[u\left(-T_{-}^{0}\right)-\dot{q}_{-}\left(-T_{-}^{0}, 0\right) T-R^{\prime}(q(0,0)) \frac{\partial q_{-}}{\partial \alpha}(0,0) \theta\right] \\
& +\psi_{1} G^{\prime}\left(q_{-}\left(-T_{-}^{0}, 0\right)\right)\left[u\left(-T_{-}^{0}\right)-\dot{q}_{-}\left(-T_{-}^{0}, 0\right) T\right]+\psi_{2} T=0
\end{aligned}
$$


or

$$
\begin{aligned}
& \left\{\left[\frac{\partial q_{-}}{\partial \alpha}(0,0)\right]^{*}\left[v(0)-R^{\prime}\left(q_{-}(0,0)\right)^{*} \psi\right]\right\}^{*} \theta \\
& \quad+\left[\psi-v\left(-T_{-}^{0}\right)+\psi_{1} G^{\prime}\left(q_{-}\left(-T_{-}^{0}, 0\right)\right)^{*}\right]^{*} u\left(-T_{-}^{0}\right) \\
& \quad-\int_{-T_{-}^{0}}^{0}\left[\dot{v}(t)+f_{-}^{\prime}\left(q_{-}(t, 0)\right)^{*} v(t)\right]^{*} u(t) d t \\
& \quad+\left[\psi_{2}-\psi^{*} \dot{q}_{-}\left(-T_{-}^{0}, 0\right)-\psi_{1} G^{\prime}\left(q_{-}\left(-T_{-}^{0}, 0\right)\right) \dot{q}_{-}\left(-T_{-}^{0}, 0\right)\right] T=0
\end{aligned}
$$

because of the definition of $\psi_{2}$ and the fact that $\left(v(t), \psi, \psi_{1}\right)$ satisfies (4.6).

To prove the sufficiency, we show that if $(h, d, T) \in Y$ does not belong to $\mathcal{R} A$, then there exists a solution of the variational equation (4.6) such that (4.7) does not hold. So, assume that $\left(h, d, 0, T_{1}\right) \notin \mathcal{R} A$. By Lemma 4.1 and the Hahn-Banach theorem, there is an $\left(\bar{v}, \bar{\psi}, \bar{\psi}_{1}, \bar{\psi}_{2}\right) \in Y$ such that

$$
\left\langle\left(\bar{v}, \bar{\psi}, \bar{\psi}_{1}, \bar{\psi}_{2}\right), A(u, \theta, T)\right\rangle=0, \quad \forall(u, \theta, T) \in X
$$

and

$$
\left\langle\left(\bar{v}, \bar{\psi}, \bar{\psi}_{1}, \bar{\psi}_{2}\right),\left(h, d, 0, T_{1}\right)\right\rangle=1 \text {, }
$$

where $\langle\cdot, \cdot\rangle$ is the usual scalar product on $Y$. We already noted that we can assume that $\bar{\psi}^{\prime \prime} \dot{q}_{+}\left(T_{+}^{0}, 0\right)=0$, and (4.8)-(4.9) remain valid. Repeating our previous arguments, we see that $v(t) \in W^{1,2}\left(\left[-T_{-}^{0}, 0\right], \mathbb{R}^{n}\right)$ and that $(4.8)$ implies $\left(\bar{v}, \bar{\psi}, \bar{\psi}_{1}, \bar{\psi}_{2}\right)$ solves the adjoint system (4.6). Summarizing, if $\left(h, d, 0, T_{1}\right) \notin \mathcal{R} A$ there exists a solution of the adjoint system for which (4.6) does not hold. This finishes the proof.

Again we note that equation (4.6) only depends on the derivative $R^{\prime}\left(q_{-}(0,0)\right)$ on $T_{q_{-}(0,0)} S$ since $\frac{\partial q_{-}}{\partial \alpha}(0,0)^{*} R^{\prime}\left(q_{-}(0,0)\right)^{*} \psi=\left[\dot{q}_{+}\left(T_{+}^{0}, 0\right) T_{+}^{\prime}(0)+\frac{\partial q_{+}}{\partial \alpha}\left(T_{+}^{0}, 0\right)\right]^{*} \psi=\frac{\partial q_{+}}{\partial \alpha}\left(T_{+}^{0}, 0\right)^{*} \psi$, where we use $\psi^{*} \dot{q}_{+}\left(T_{+}^{0}, 0\right)=0$ or, in other words, it is independent of any $C^{1}$-extension we take of $R(\xi)$ to the whole $U_{0}$.

We now prove the following proposition.

Proposition 4.2 The adjoint system (4.6) has a solution if and only if ( $\left.\psi, \psi_{1}\right)$ satisfy the first and the third equation in (3.1) (and we take the second equation in (3.1) as definition of $\left.\psi_{2}\right)$.

Proof Indeed let $v(t)$ be a solution of (4.6) then

$$
v(t)=Y(t) Y\left(-T_{-}^{0}\right)^{-1} v\left(-T_{-}^{0}\right)
$$

$Y(t)=X_{-}^{-1}(t)^{*}$ being the fundamental matrix of the linear equation $\dot{v}(t)+f_{-}^{\prime}\left(q_{-}(t, 0)\right)^{*} \times$ $v(t)=0$. Then, taking $v\left(-T_{-}^{0}\right)=\psi+\psi_{1} G^{\prime}\left(q_{-}\left(-T_{-}^{0}, 0\right)\right)^{*}$ the two remaining condition in (4.6) read:

$$
\left\{\begin{array}{l}
{\left[\frac{\partial q_{-}}{\partial \alpha}(0,0)\right]^{*}\left[Y\left(-T_{-}^{0}\right)^{-1}\left[\psi+\psi_{1} G^{\prime}\left(q_{-}\left(-T_{-}^{0}, 0\right)\right)^{*}\right]-R^{\prime}\left(q_{-}(0,0)^{*} \psi\right]=0\right.} \\
\psi^{*} \dot{q}_{+}\left(T_{+}^{0}, 0\right)=0
\end{array}\right.
$$


that can be written as

$$
\left\{\begin{array}{l}
\frac{\partial q_{-}}{\partial \alpha}\left(-T_{-}^{0}, 0\right)^{*}\left[\psi+\psi_{1} G^{\prime}\left(q_{-}\left(-T_{-}^{0}, 0\right)\right)^{*}\right]-\left[\psi^{\prime \prime} R^{\prime}\left(q_{-}(0,0) \frac{\partial q_{-}}{\partial \alpha}(0,0)\right]^{*}=0\right. \\
\psi^{*} \dot{q}_{+}\left(T_{+}^{0}, 0\right)=0
\end{array}\right.
$$

or else, on account of $R\left(q_{-}(0, \alpha)\right)=q_{+}\left(T_{+}(\alpha), \alpha\right)$ :

$$
\left\{\begin{array}{l}
\psi^{*}\left[\frac{\partial q_{-}}{\partial \alpha}\left(-T_{-}^{0}, 0\right)-\frac{\partial q_{+}}{\partial \alpha}\left(T_{+}^{0}, 0\right)\right]+\psi_{1} G^{\prime}\left(q_{-}\left(-T_{-}^{0}, 0\right)\right) \frac{\partial q_{-}}{\partial \alpha}\left(-T_{-}^{0}, 0\right)=0, \\
\psi^{\prime \prime} \dot{q}_{+}\left(T_{+}^{0}, 0\right)=0 .
\end{array}\right.
$$

The proof is finished.

We conclude this section giving another expression of the Poincaré-Melnikov function (3.2) in terms of the solution of the adjoint system (4.6). To this end, let $v(t)$ be a solution of the adjoint system (4.6). Since a fundamental matrix of the linear equation

$$
\dot{v}+f_{-}^{\prime}\left(q_{-}(t, 0)\right) v=0
$$

is $X_{-}^{-1}(t)^{\prime \prime}$ we see that

$$
v(t)=X_{-}^{-1}(t)^{*} X_{-}\left(-T_{-}^{0}\right)^{*} v\left(-T_{-}^{0}\right)=X_{-}^{-1}(t)^{*} X_{-}\left(-T_{-}^{0}\right)^{*}\left[\psi+\psi_{1} G^{\prime}\left(q_{-}\left(-T_{-}^{0}, 0\right)\right)^{*}\right]
$$

so:

$$
v^{*}(t)=\left[\psi^{*}+\psi_{1} G^{\prime}\left(q_{-}\left(-T_{-}^{0}, 0\right)\right)\right] X_{-}\left(-T_{-}^{0}\right) X_{-}^{-1}(t)
$$

Then

$$
\mathcal{M}(\tau)=\psi^{\prime \prime} \int_{0}^{T_{+}^{0}} X_{+}\left(T_{+}^{0}\right) X_{+}(t)^{-1} g_{+}\left(\tau, q_{+}(0,0), 0\right) d t+\int_{-T_{-}^{0}}^{0} v(t)^{*} g_{-}\left(t+\tau, q_{-}(t, 0), 0\right) d t .
$$

As for the first term in the above equality, we can show it is related to the impact $R(\tau ; \xi, \varepsilon)$. Indeed, from Section 2 we know that the solution of the singular equation

$$
\dot{x}=f_{+}(x)+\varepsilon g(t, x, \varepsilon)
$$

can be written as

$$
x(t+\tau)=x_{+}(t)+q_{+}\left(\varepsilon^{-1} t, \alpha\right)
$$

with $x_{+}(\varepsilon t)$ as in equation (2.6). Thus, $\xi=x(\tau)=q_{+}(0, \alpha) \in S$ and

$$
\begin{aligned}
& R(\tau ; \xi, \varepsilon)=x_{+}\left(\varepsilon T_{+}\right)+q_{+}\left(T_{+}, \alpha\right) \\
& \quad=\int_{0}^{T_{+}} X_{+}\left(T_{+}, \alpha\right) X_{+}^{-1}(s, \alpha) h_{+}\left(\varepsilon s, \tau, x_{+}(\varepsilon s), \alpha, \varepsilon\right) d s+q_{+}\left(T_{+}, \alpha\right)
\end{aligned}
$$


for some $T_{+}=T_{+}(\tau ; \alpha, \varepsilon)$. Then

$$
\frac{\partial R}{\partial \varepsilon}\left(\tau ; q_{-}(0,0), 0\right)=\dot{q}_{+}\left(T_{+}^{0}, 0\right) \frac{\partial T_{+}}{\partial \varepsilon}+\int_{0}^{T_{+}^{0}} X_{+}\left(T_{+}^{0}\right) X_{+}^{-1}(s) g_{+}\left(\tau, q_{+}(0,0), 0\right) d s
$$

and then, using again $\psi^{\prime \prime} \dot{q}_{+}\left(T_{+}^{0}, 0\right)=0$ we see that

$$
\psi^{*} \int_{0}^{T_{+}^{0}} X_{+}\left(T_{+}^{0}\right) X_{+}^{-1}(s) g_{+}\left(\tau, q_{+}(0,0), 0\right) d s=\psi \frac{\partial R}{\partial \varepsilon}\left(\tau ; q_{-}(0,0), 0\right)
$$

i.e.

$$
\mathcal{M}(\tau)=\psi^{\prime \prime} \frac{\partial R}{\partial \varepsilon}\left(\tau ; q_{-}(0,0), 0\right)+\int_{-T_{-}^{0}}^{0} v(t)^{*} g_{-}\left(t+\tau, q_{-}(t, 0), 0\right) d t .
$$

When $g_{+}$is autonomous, then $R$ is independent of $\tau$, and the expression (4.10) of the Poincaré-Melnikov function should be compared with the one given in [14, Theorem 4.2] where a Poincaré-Melnikov function, characterizing transition to chaos, is given for almost periodic perturbations of autonomous impact equations with a homoclinic orbit.

\section{The case of a manifold of periodic solutions}

In this section we assume that $q_{-}\left(-T_{-}(\alpha), \alpha\right)=q_{+}\left(T_{+}(\alpha), \alpha\right)$ for any $\alpha$ in (an open neighborhood of $\alpha=0$ in) $\mathbb{R}^{n-1}$. Hence, from (3.3), we see that

$$
\mathcal{F}\left(T_{+}(\alpha), T_{-}(\alpha), \tau, \alpha, 0\right)=\left(\begin{array}{c}
0 \\
0 \\
T_{-}(\alpha)-T_{-}^{0}
\end{array}\right)
$$

We distinguish the two cases: $T_{-}^{\prime}(0) \neq 0$ and $T_{-}(\alpha)=T_{-}^{0}$ for all $\alpha$ in (an open neighborhood of $\alpha=0$ in) $\mathbb{R}^{n-1}$. First, we assume that

$$
T_{-}^{\prime}(0) \neq 0 .
$$

Then a $C^{2},(n-2)$-dimensional submanifold $\mathcal{S}$ of (an open neighborhood of $\alpha=0$ in) $\mathbb{R}^{n-1}$ exists such that $T_{-}(\alpha)=T_{-}^{0}$ for any $\alpha \in \mathcal{S}$. So, for $\varepsilon=0, \mathcal{F}\left(T_{+}, T_{-}, \tau, \alpha, 0\right)=0$ has the $(n-1)$-dimensional manifold of solutions

$$
\left(T_{+}, T_{-}, \tau, \alpha\right)=\xi(\alpha, \tau):=\left(T_{+}(\alpha), T_{-}^{0}, \tau, \alpha\right), \quad(\alpha, \tau) \in \mathcal{S} \times \mathbb{R} .
$$

So, we are in position to apply Theorem 3.2. First, we have to verify that the kernel $\mathcal{N} D_{1} \mathcal{F}(\xi(\alpha, \tau), 0)$ equals the tangent space $T_{\xi(\alpha, \tau)} \mathcal{X}, \mathcal{X}=\{\xi(\alpha, \tau) \mid(\alpha, \tau) \in \mathcal{S} \times \mathbb{R}\}$, and then that the Poincaré-Melnikov function (vector):

$$
[\mathbb{I}-\Pi(\alpha, \tau)] D_{2} \mathcal{F}(\xi(\alpha, \tau), 0)
$$


has a simple zero at $(\alpha, \tau)=\left(0, \tau_{0}\right)$. Note that

$$
T_{\xi(\alpha, \tau)} \mathcal{X}=\operatorname{span}\left\{\left(\begin{array}{c}
T_{+}^{\prime}(\alpha) v \\
0 \\
0 \\
v
\end{array}\right),\left(\begin{array}{l}
0 \\
0 \\
1 \\
0
\end{array}\right): v \in T_{\alpha} \mathcal{S}\right\}
$$

From (3.3), we get:

$$
\begin{array}{rl}
D_{1} & \mathcal{F}(\xi(\alpha, \tau), 0) \\
\quad=\left(\begin{array}{cccc}
-\dot{q}_{+}\left(T_{+}(\alpha), \alpha\right) & -\dot{q}_{-}\left(-T_{-}^{0}, \alpha\right) & 0 & \frac{\partial q_{-}}{\partial \alpha}\left(-T_{-}^{0}, \alpha\right)-\frac{\partial q_{+}}{\partial \alpha}\left(T_{+}(\alpha), \alpha\right) \\
0 & -G^{\prime}\left(q_{-}\left(-T_{-}^{0}, \alpha\right)\right) \dot{q}_{-}\left(-T_{-}^{0}, \alpha\right) & 0 & G^{\prime}\left(q_{-}\left(-T_{-}^{0}, \alpha\right)\right) \frac{\partial q_{-}}{\partial \alpha}\left(-T_{-}^{0}, \alpha\right) \\
0 & 1 & 0 & 0
\end{array}\right) .
\end{array}
$$

Note that $D_{1} \mathcal{F}(\xi(\alpha, \tau), 0)$ does not depend on $\tau$. Using $G\left(q_{-}\left(-T_{-}^{0}, \alpha\right)\right)=0$ and $q_{-}\left(-T_{-}^{0}, \alpha\right)=$ $q_{+}\left(T_{+}(\alpha), \alpha\right)$ for any $\alpha \in \mathcal{S}$ we easily see that

$$
D_{1} \mathcal{F}(\xi(\alpha, \tau), 0)_{\left.\right|_{T_{\xi(\alpha, \tau)} \mathcal{X}}}=0
$$

for any $v \in T_{\alpha} \mathcal{S}$. On the other hand, assume that

$$
\begin{aligned}
& \left(\begin{array}{c}
\mu_{+} \\
\mu_{-} \\
w
\end{array}\right) \\
& \quad \in \mathcal{N}\left(\begin{array}{ccc}
-\dot{q}_{+}\left(T_{+}(\alpha), \alpha\right) & -\dot{q}_{-}\left(-T_{-}^{0}, \alpha\right) & \frac{\partial q_{-}}{\partial \alpha}\left(-T_{-}^{0}, \alpha\right)-\frac{\partial q_{+}}{\partial \alpha}\left(T_{+}(\alpha), \alpha\right) \\
0 & -G^{\prime}\left(q_{-}\left(-T_{-}^{0}, \alpha\right)\right) \dot{q}_{-}\left(-T_{-}^{0}, \alpha\right) & G^{\prime}\left(q_{-}\left(-T_{-}^{0}, \alpha\right)\right) \frac{\partial q_{-}}{\partial \alpha}\left(-T_{-}^{0}, \alpha\right) \\
0 & 1 & 0
\end{array}\right)
\end{aligned}
$$

for some $\mu_{+}, \mu_{-} \in \mathbb{R}$ and $w \in \mathbb{R}^{n-1}$. Then $\mu_{-}=0$ and $\left(\mu_{+}, w\right)$ satisfies

$$
\left\{\begin{array}{l}
-\dot{q}_{+}\left(T_{+}(\alpha), \alpha\right) \mu_{+}+\left[\frac{\partial q_{-}}{\partial \alpha}\left(-T_{-}^{0}, \alpha\right)-\frac{\partial q_{+}}{\partial \alpha}\left(T_{+}(\alpha), \alpha\right)\right] w=0, \\
G^{\prime}\left(q_{-}\left(-T_{-}^{0}, \alpha\right)\right) \frac{\partial q_{-}}{\partial \alpha}\left(-T_{-}^{0}, \alpha\right) w=0
\end{array}\right.
$$

that, on account of $q_{-}\left(-T_{-}^{0}, \alpha\right)=q_{+}\left(T_{+}(\alpha), \alpha\right)$ is equivalent to

$$
\left\{\begin{array}{l}
\dot{q}_{+}\left(T_{+}(\alpha), \alpha\right)\left[T_{+}^{\prime}(\alpha) w-\mu_{+}\right]=0, \\
G^{\prime}\left(q_{-}\left(-T_{-}^{0}, \alpha\right)\right) \frac{\partial q_{-}}{\partial \alpha}\left(-T_{-}^{0}, \alpha\right) w=0
\end{array}\right.
$$

Now, from $G\left(q_{-}\left(-T_{-}(\alpha), \alpha\right)\right)=0$ we get, for any $w \in \mathbb{R}^{n-1}$ :

$$
G^{\prime}\left(q_{-}\left(-T_{-}(\alpha), \alpha\right)\right) \frac{\partial q_{-}}{\partial \alpha}\left(-T_{-}(\alpha), \alpha\right) w=G^{\prime}\left(q_{-}\left(-T_{-}(\alpha), \alpha\right)\right) \dot{q}_{-}\left(-T_{-}(\alpha), \alpha\right) T_{-}^{\prime}(\alpha) w
$$

and hence

$$
G^{\prime}\left(q_{-}\left(-T_{-}^{0}, \alpha\right)\right) \frac{\partial q_{-}}{\partial \alpha}\left(-T_{-}, \alpha\right) w=0 \quad \Leftrightarrow \quad G^{\prime}\left(q_{-}\left(-T_{-}^{0}, \alpha\right)\right) \dot{q}_{-}\left(-T_{-}^{0}, \alpha\right) T_{-}^{\prime}(\alpha) w=0
$$


which, in turn, is equivalent to $w \in T_{\alpha} \mathcal{S}$ because of transversality and the fact that $T_{\alpha} \mathcal{S}=$ $\mathcal{N} T_{-}^{\prime}(\alpha)$.

Hence, we conclude that $\mathcal{N} D_{1} \mathcal{F}(\xi(\alpha, \tau), 0)=T_{\xi(\alpha, \tau)} \mathcal{X}$.

Now we consider the second condition. The Poincaré-Melnikov function (vector) [II$\Pi(\alpha, \tau)] D_{2} \mathcal{F}(\xi(\alpha, \tau), 0), \alpha \in \mathcal{S}$ can be written as

$$
\psi^{*}(\alpha, \tau) D_{2} \mathcal{F}(\xi(\alpha, \tau), 0)
$$

where $\psi^{*}(\alpha, \tau)$ is a matrix whose rows are left eigenvectors of zero eigenvalue of the matrix $D_{1} \mathcal{F}(\xi(\alpha, \tau), 0)$, that is,

$$
\psi^{*}(\alpha, \tau) D_{1} \mathcal{F}(\xi(\alpha, \tau), 0)=0
$$

Note that $\psi(\alpha, \tau)=\psi(\alpha)$ does not depend on $\tau$ since so does $D_{1} \mathcal{F}(\xi(\alpha, \tau), 0)$. Then (5.1) reads:

$$
\begin{aligned}
\mathcal{M}(\alpha, \tau):= & \psi^{*}(\alpha) \int_{0}^{T_{+}^{0}} X_{+}\left(T_{+}^{0}, \alpha\right) X_{+}(s, \alpha)^{-1} g_{+}(\tau, q(0, \alpha), 0) d s \\
& +\psi^{\prime \prime}(\alpha) \int_{-T_{-}^{0}}^{0} X_{-}\left(-T_{-}^{0}, \alpha\right) X_{-}(s, \alpha)^{-1} g_{-}\left(s+\tau, q_{-}(s, \alpha), 0\right) d s \\
& +\psi_{1}(\alpha) G^{\prime}\left(q\left(-T_{-}^{0}, \alpha\right)\right) \int_{-T_{-}^{0}}^{0} X_{-}\left(-T_{-}^{0}, \alpha\right) X_{-}^{-1}(s, \alpha) g_{-}\left(s+\tau, q_{-}(s, \alpha), 0\right) d s .
\end{aligned}
$$

Arguing as in Section 3, equation (5.2) is equivalent to

$$
\left\{\begin{array}{l}
\psi^{*}(\alpha) \dot{q}_{+}\left(T_{+}(\alpha), \alpha\right)=0, \\
\psi_{2}(\alpha)=\left[\psi^{*}(\alpha)+\psi_{1}(\alpha) G^{\prime}\left(q\left(-T_{-}^{0}, \alpha\right)\right)\right] \dot{q}_{-}\left(-T_{-}^{0}, \alpha\right), \\
\psi^{\prime \prime}(\alpha)\left[\frac{\partial q_{-}}{\partial \alpha}\left(-T_{-}^{0}, \alpha\right)-\frac{\partial q_{+}}{\partial \alpha}\left(T_{+}(\alpha), \alpha\right)\right]+\psi_{1}(\alpha) G^{\prime}\left(q\left(-T_{-}^{0}, \alpha\right)\right) \frac{\partial q_{-}}{\partial \alpha}\left(-T_{-}^{0}, \alpha\right)=0 .
\end{array}\right.
$$

Moreover, the adjoint variational system along $q_{-}(t, \alpha)$ is defined as

$$
\left\{\begin{array}{l}
\dot{v}(t)+f_{-}^{\prime}\left(q_{-}(t, \alpha)\right)^{*} v(t)=0, \\
{\left[\frac{\partial q_{-}}{\partial \alpha}(0, \alpha)\right]^{*}\left[v(0)-R^{\prime}\left(q_{-}(0, \alpha)\right)^{*} \psi(\alpha)\right]=0,} \\
v\left(-T_{-}^{0}\right)=\psi(\alpha)+\psi_{1}(\alpha) G^{\prime}\left(q_{-}\left(-T_{-}^{0}, \alpha\right)\right)^{*}, \\
\psi^{*}(\alpha) \dot{q}_{+}\left(T_{+}(\alpha), \alpha\right)=0,
\end{array}\right.
$$

where $\left(\psi^{*}(\alpha), \psi_{1}(\alpha), \psi_{2}(\alpha)\right)$ satisfy equation (5.2). Then the Poincaré-Melnikov vector can be written as

$$
\begin{aligned}
\mathcal{M}(\alpha, \tau)= & \psi^{*}(\alpha) \int_{0}^{T_{+}(\alpha)} X_{+}\left(T_{+}(\alpha), \alpha\right) X_{+}(t, \alpha) g_{+}\left(\tau, q_{+}(0, \alpha), 0\right) d t \\
& +\int_{-T_{-}^{0}}^{0} v(t, \alpha)^{*} g_{-}\left(t+\tau, q_{-}(t, \alpha), 0\right) d t
\end{aligned}
$$

or else

$$
\mathcal{M}(\alpha, \tau)=\psi^{*}(\alpha) \frac{\partial R}{\partial \varepsilon}\left(\tau ; q_{-}(0, \alpha), 0\right)+\int_{-T_{-}^{0}}^{0} v(t, \alpha)^{*} g_{-}\left(t+\tau, q_{-}(t, \alpha), 0\right) d t
$$


$v(t, \alpha)$ being the solution of (5.4) and $X_{+}(t, \alpha)$ the fundamental matrix of the linear equation

$$
\dot{x}=f_{+}^{\prime}\left(q_{+}(t, \alpha)\right) x .
$$

Of course the only difference between the cases $T_{-}^{\prime}(0) \neq 0$ and $T_{-}(\alpha)=T_{-}^{0}$ for all $\alpha \in S$ is that in the first case the Poincaré-Melnikov function is defined for $(\alpha, \tau) \in \mathcal{S} \times \mathbb{R}$ while in the second it is defined for $(\alpha, \tau) \in \mathcal{O} \times \mathbb{R}$ for an open neighborhood $\mathcal{O}$ of $0 \in \mathbb{R}^{n-1}$. Summarizing, we proved the following result.

Theorem 5.1 Assume that $q_{-}\left(-T_{-}(\alpha), \alpha\right)=q_{+}\left(T_{+}(\alpha), \alpha\right)$ for any $\alpha$ in a neighborhood of $\alpha=0$, and that either $T_{-}^{\prime}(0) \neq 0$ or $T_{-}(\alpha)=T_{-}^{0}$ for any $\alpha$ (in the same neighborhood). Then system (5.3) has a $d$-dimensional space of solutions where $d=n$ or $d=n+1$ according to which of the two conditions $T_{-}^{\prime}(0) \neq 0$ or $T_{-}(\alpha)=T_{-}^{0}$ holds. Moreover, if the PoincaréMelnikov function (5.5) (or (5.6)) has a simple zero at $\left(0, \tau_{0}\right)$ then system (1.1) has a $T_{\varepsilon}$ periodic solution $x(t, \varepsilon)$ satisfying (2.1).

Finally, we note that when we can show that a Brouwer degree of a Poincaré-Melnikov function from either Theorem 3.1 or 5.1 is non-zero then by following [15] we can show existence results.

\section{Examples}

We consider a second-order equation

$$
\begin{cases}\varepsilon^{2} \ddot{x}=f_{+}(x, \dot{x})+\varepsilon g_{+}(t, x, \dot{x}, \varepsilon), & x>0, \\ \ddot{x}=f_{-}(x)+\varepsilon g_{-}(t, x, \dot{x}, \varepsilon), & x<0\end{cases}
$$

with the line $x=0$ as discontinuity manifold (i.e., with $G(x, \dot{x})=x$ ). We write $q_{ \pm}(t, \alpha)=$ $\left(\begin{array}{l}q_{1}^{ \pm}(t, \alpha) \\ \dot{q}_{1}^{ \pm}(t, \alpha)\end{array}\right)$ with $q_{-}(0, \alpha)=\left(\begin{array}{c}0 \\ \alpha+\alpha_{0}\end{array}\right)$ (i.e. $q_{1}^{ \pm}(0, \alpha)=0$ and $\left.\dot{q}_{1}^{-}(0, \alpha)=\alpha+\alpha_{0}\right)$. We also write $q_{+}\left(T_{+}(\alpha), \alpha\right)=\left(\begin{array}{c}0 \\ \varphi(\alpha)\end{array}\right)$ so that

$$
R:\left(\begin{array}{c}
0 \\
\alpha+\alpha_{0}
\end{array}\right) \mapsto\left(\begin{array}{c}
0 \\
\varphi(\alpha)
\end{array}\right)
$$

i.e., we take

$$
R\left(x_{1}, x_{2}\right)=\left(\begin{array}{c}
0 \\
\varphi\left(x_{2}-\alpha_{0}\right)
\end{array}\right)
$$

in the plane coordinates $\left(x_{1}, x_{2}\right)$. According to equation (5.4), the adjoint variational system reads, with $\psi(\alpha)=\left(\begin{array}{l}\psi^{\prime}(\alpha) \\ \psi^{\prime \prime}(\alpha)\end{array}\right)$ :

$$
\left\{\begin{array}{l}
\dot{v}_{1}=-f_{-}^{\prime}\left(q_{1}^{-}(t, \alpha)\right) v_{2}, \\
\dot{v}_{2}=-v_{1}, \\
v_{2}(0)-\varphi^{\prime}(\alpha) \psi^{\prime \prime}(\alpha)=0, \\
v_{1}\left(-T_{-}^{0}\right)=\psi^{\prime}(\alpha)+\psi_{1}(\alpha), \\
v_{2}\left(-T_{-}^{0}\right)=\psi^{\prime \prime}(\alpha), \\
\psi^{\prime}(\alpha) \varphi(\alpha)+\psi^{\prime \prime}(\alpha) f_{+}(0, \varphi(\alpha))=0
\end{array}\right.
$$


which can be written as (with $v_{2}=w$ and $v_{1}=-\dot{w}$ ):

$$
\left\{\begin{array}{l}
\ddot{w}=f_{-}^{\prime}\left(q_{1}^{-}(t, \alpha)\right) w, \\
w(0)-\varphi^{\prime}(\alpha) w\left(-T_{-}^{0}\right)=0, \\
\psi^{\prime \prime}=w\left(-T_{-}^{0}\right), \\
\psi^{\prime} \varphi(\alpha)+\psi^{\prime \prime} f_{+}(0, \varphi(\alpha))=0, \\
\psi_{1}=-\dot{w}\left(-T_{-}^{0}\right)-\psi^{\prime} .
\end{array}\right.
$$

Note that (when $\varphi(\alpha) \neq 0$ ) the last three equation are actually the definitions of $\psi(\alpha)=$ $\left(\begin{array}{l}\psi^{\prime}(\alpha) \\ \psi^{\prime \prime}(\alpha)\end{array}\right)$, and $\psi_{1}(\alpha)$ in terms of the unique (up to a multiplicative constant) bounded solution of the boundary value problem:

$$
\left\{\begin{array}{l}
\ddot{w}=f_{-}^{\prime}\left(q_{1}^{-}(t, \alpha)\right) w \\
w(0)-\varphi^{\prime}(\alpha) w\left(-T_{-}^{0}\right)=0
\end{array}\right.
$$

and the Poincaré-Melnikov function (5.6) reads:

$$
\begin{aligned}
\mathcal{M}(\alpha, \tau)= & w\left(-T_{-}^{0}, \alpha\right)\left(-\frac{f_{+}(0, \varphi(\alpha))}{\varphi(\alpha)} \quad 1\right) \frac{\partial R}{\partial \varepsilon}\left(\tau ; q_{-}(0, \alpha), 0\right) \\
& +\int_{-T_{-}^{0}}^{0} w(t, \alpha) g_{-}\left(t+\tau, q_{-}(t, \alpha), 0\right) d t
\end{aligned}
$$

whereas (4.10) reads:

$$
\mathcal{M}(\tau)=w\left(-T_{-}^{0}\right)\left(-\frac{f_{+}(0, \varphi(0))}{\varphi(0)} \quad \text { 1) } \frac{\partial R}{\partial \varepsilon}\left(\tau ; q_{-}(0,0), 0\right)+\int_{-T_{-}^{0}}^{0} w(t) g_{-}\left(t+\tau, q_{-}(t, 0), 0\right) d t .\right.
$$

As an example, we take $f_{-}(x)=-x$ that is we consider the equation

$$
\ddot{x}+x=\varepsilon g_{-}(t, x, \dot{x}, \varepsilon) .
$$

The unperturbed equation $\ddot{x}+x=0$ with the condition $\dot{x}(0)=0$ has the solutions:

$$
q_{-}(t, \alpha)=\left(\alpha+\alpha_{0}\right)\left(\begin{array}{c}
\sin t \\
\cos t
\end{array}\right), \quad-\pi \leq t \leq 0
$$

and $T_{-}(\alpha)=\pi$. Note that, to have $q_{-}(t, \alpha) \in\left\{\left(x_{1}, x_{2}\right) \mid x_{1}<0\right\}$ for $-\pi<t<0$ we need $\alpha+$ $\alpha_{0}>0$

We assume we are in the first (non degenerate) case that is it holds (1.4), which now has the form

$$
\begin{aligned}
& R\left(q_{-}(0,0)\right)=q_{-}(-\pi, 0), \\
& \frac{\partial}{\partial \alpha}\left[R\left(q_{-}(0, \alpha)\right)-q_{-}(-\pi, \alpha)\right]_{\alpha=0} \neq 0 .
\end{aligned}
$$

Note $T_{-}^{\prime}(0)=0$ for this case. Since

$$
R\left(q_{-}(0, \alpha)\right)-q_{-}(-\pi, \alpha)=\left(\begin{array}{c}
0 \\
\varphi(\alpha)+\alpha+\alpha_{0}
\end{array}\right),
$$


(6.2) is equivalent to

$$
\varphi(0)=-\alpha_{0}, \quad \varphi^{\prime}(0)+1 \neq 0 .
$$

Then it is easily seen that system (6.1), with $\alpha=0$, reads

$$
\left\{\begin{array}{l}
\ddot{w}+w=0, \\
w(0)-\varphi^{\prime}(0) w(-\pi)=0, \\
\psi^{\prime \prime}=w(-\pi), \\
-\psi^{\prime} \alpha_{0}+\psi^{\prime \prime} f_{+}\left(0,-\alpha_{0}\right)=0, \\
\psi_{1}=-\dot{w}(-\pi)-\psi^{\prime} .
\end{array}\right.
$$

Solving $\ddot{w}+w=0$, we get $w(t)=a \cos \left(t+t_{0}\right)$ and the boundary condition reads: $a(1+$ $\left.\varphi^{\prime}(0)\right) \cos t_{0}=0$. So, we can take $w(t)=\cos \left(t-\frac{\pi}{2}\right)=\sin t$. Since $\varphi(0)=-\alpha_{0} \neq 0$, then

$$
\psi^{\prime \prime}=0, \quad \psi^{\prime}=0, \quad \psi_{1}=1
$$

and the Poincaré-Melnikov function reads

$$
\mathcal{M}(\tau)=\int_{-\pi}^{0} g_{-}\left(t+\tau, \alpha_{0} \sin t, \alpha_{0} \cos t, 0\right) \sin t d t .
$$

For example, taking $g_{-}(t, x, \dot{x}, \varepsilon)=-\dot{x} \cos ^{2}\left(\frac{\pi}{\pi+\varepsilon T_{+}^{0}}\right) t$, where $T_{+}^{0}$ is the time the solution of equation $\ddot{x}=f_{+}(x, \dot{x}), x(0)=0, \dot{x}(0)=\alpha_{0}$ takes to reach the discontinuity manifold $x=0$, we get

$$
\mathcal{M}(\tau)=\frac{\pi}{8} \alpha_{0} \sin (2 \tau)
$$

which has a simple zero at $\tau=0$.

To conclude the example we need to find a second-order equation $\ddot{x}=f_{+}(x, \dot{x})$ such that (6.3) holds. We consider

$$
\ddot{x}+x=f_{+}(x, \dot{x}):=f\left(x^{2}+\dot{x}^{2}-1\right) g(x, \dot{x})
$$

with $f(0)=0$ and $f^{\prime}(0) \neq 0$. It has the solution $x=\sin t$ and $y=\cos t$. So, we take $q_{+}(t)=$ $(\sin t, \cos t)$ and then $T_{+}^{0}=\pi$. Note $q_{+}\left(T_{+}(\alpha), \alpha\right)=\left(\begin{array}{c}0 \\ \varphi(\alpha)\end{array}\right)$ is equivalent to $q_{1}^{+}\left(T_{+}(\alpha), \alpha\right)=0$ and $\dot{q}_{1}^{+}\left(T_{+}(\alpha), \alpha\right)=\varphi(\alpha)$. Then $\varphi(0)=-1$, so we take $\alpha_{0}=1$. Furthermore,

$$
\varphi^{\prime}(0)=\ddot{q}_{1}^{+}(\pi, 0) T_{+}^{\prime}(0)+\frac{\partial}{\partial \alpha} \dot{q}_{1}^{+}(\pi, 0)=\frac{\partial}{\partial \alpha} \dot{q}_{1}^{+}(\pi, 0) .
$$

Setting $\zeta(t):=\frac{\partial}{\partial \alpha} q_{1}^{+}(t, 0)$, we have

$$
\ddot{\zeta}+\zeta=2 f^{\prime}(0) g(\sin t, \cos t)(\zeta \sin t+\dot{\zeta} \cos t)
$$


Since $q_{1}^{+}(0, \alpha)=0$ and $\dot{q}_{1}^{+}(0, \alpha)=\alpha+1$, we obtain $\zeta(0)=0$ and $\dot{\zeta}(0)=1$. Clearly, (6.4) has a solution $\zeta_{1}(t)=\cos t$. Then the second solution is

$$
\begin{aligned}
\zeta_{2}(t)= & \cos t \int_{0}^{t} \frac{\mathrm{e}^{2 f^{\prime}(0) \int_{0}^{s} \cos \sigma g(\sin \sigma, \cos \sigma) d \sigma}}{\cos ^{2} s} d s=\sin t \mathrm{e}^{2 f^{\prime}(0) \int_{0}^{t} \cos \sigma g(\sin \sigma, \cos \sigma) d \sigma} \\
& +2 f^{\prime}(0) \cos t \int_{0}^{t} g(\sin s, \cos s) \mathrm{e}^{2 f^{\prime}(0) \int_{0}^{s} \cos \sigma g(\sin \sigma, \cos \sigma) d \sigma} \sin s d s
\end{aligned}
$$

Hence,

$$
\begin{aligned}
\dot{\zeta}_{2}(t)= & \cos t \mathrm{e}^{2 f^{\prime}(0) \int_{0}^{t} g(\sin \sigma, \cos \sigma) \cos \sigma d v} \\
& +f^{\prime}(0) \sin 2 \operatorname{tg}(\sin t, \cos t) \mathrm{e}^{2 f^{\prime}(0) \int_{0}^{t} g(\sin \sigma, \cos \sigma) \cos \sigma d \sigma} \\
& -2 f^{\prime}(0) \sin t \int_{0}^{t} \sin s g(\sin s, \cos s) \mathrm{e}^{2 f^{\prime}(0) \int_{0}^{s} g(\sin \sigma, \cos \sigma) \cos \sigma d \sigma} d s \\
& +f^{\prime}(0) \sin 2 \operatorname{tg}(\sin t, \cos t) \mathrm{e}^{2 f^{\prime}(0) \int_{0}^{t} g(\sin \sigma, \cos \sigma) \cos \sigma d \sigma} .
\end{aligned}
$$

This implies

$$
\varphi^{\prime}(0)=\dot{\zeta}_{2}(\pi)=-\mathrm{e}^{2 f^{\prime}(0) \int_{0}^{\pi} g(\sin \sigma, \cos \sigma) \cos \sigma d \sigma} .
$$

Consequently, if

$$
\int_{0}^{\pi} g(\sin \sigma, \cos \sigma) \cos \sigma d \sigma \neq 0
$$

then $\varphi^{\prime}(0) \neq-1$. So, we conclude with the following.

Corollary 6.1 Let $f(r)$ and $g(x, \dot{x}), g_{-}(t, x, \dot{x}, \varepsilon)$ be $C^{2}$ functions such that $f(0)=0 \neq f^{\prime}(0)$, $g_{-}(t, x, \dot{x}, \varepsilon)=g_{-}(t+(1+\varepsilon) \pi, x, \dot{x}, \varepsilon)$ and

$$
\int_{0}^{\pi} g(\sin t, \cos t) \cos t d t \neq 0
$$

Suppose, also, that the function

$$
\mathcal{M}(\tau):=\int_{-\pi}^{0} g_{-}(t+\tau, \sin t, \cos t, 0) \sin t d t
$$

has a simple zero at $\tau=0$. Then, for $\varepsilon>0$, sufficiently small the singularly perturbed system

$$
\begin{cases}\varepsilon^{2} \ddot{x}+x=f\left(x^{2}+\dot{x}^{2}-1\right) g(x, \dot{x}) & \text { if } x>0, \\ \ddot{x}+x=\varepsilon g_{-}(t, x, \dot{x}, \varepsilon) & \text { if } x<0\end{cases}
$$

has $a(1+\varepsilon) \pi$-periodic solution orbitally near the set $\{(\sin t, \cos t) \mid-\pi \leq t \leq \pi\}$.

To get a second example, we change the above as follows: we take

$$
\Omega_{+}=\{(x, \dot{x}) \mid x<0, \dot{x}>0\} \quad \text { and } \quad \Omega_{-}=\mathbb{R}^{2} \backslash \Omega_{+}
$$


with equations:

$$
\begin{cases}\ddot{x}+x=\varepsilon g_{-}(t, x, \dot{x}, \varepsilon) & \text { for }(x, \dot{x}) \in \Omega_{-}, \\ \varepsilon^{2} \ddot{x}+2 x+\frac{3}{2} x^{2}=0 & \text { for }(x, \dot{x}) \in \Omega_{+} .\end{cases}
$$

It should be noted that the discontinuity line is the union of the two half lines $\{x=0, \dot{x}>0\}$ and $\{x<0, \dot{x}=0\}$ which is not $C^{1}$. However, all results hold true as long as we remain outside a (small) neighborhood of $(0,0)$.

The unperturbed equation on $\Omega_{-}$has the solutions:

$$
q_{-}(t, \alpha)=(\alpha+1)\left(\begin{array}{c}
-\cos t \\
\sin t
\end{array}\right), \quad-\frac{3}{2} \pi \leq t \leq 0
$$

with $q_{-}(0, \alpha)=-(\alpha+1)\left(\begin{array}{l}1 \\ 0\end{array}\right)$ and $q_{-}\left(-\frac{3}{2} \pi, \alpha\right)=(\alpha+1)\left(\begin{array}{l}0 \\ 1\end{array}\right)$. Then $q_{+}\left(T_{+}(\alpha), \alpha\right)=R\left(q_{-}(0, \alpha)\right)$ is the value of the solution $\left(\begin{array}{l}z_{+}(t, \alpha) \\ \dot{z}_{+}(t, \alpha)\end{array}\right)$ of

$$
\ddot{x}+2 x+\frac{3}{2} x^{2}=0, \quad x(0)=-(1+\alpha), \dot{x}(0)=0
$$

at the time $T_{+}(\alpha)$ where $z_{+}\left(T_{+}(\alpha), \alpha\right)=0$. Since the equation has the Hamiltonian $H_{+}(x, \dot{x})=$ $\dot{x}^{2}+(x+2) x^{2}$, we see that $z_{+}(t, \alpha)$ satisfies

$$
\dot{z}^{2}(t)+(z(t)+2) z^{2}(t)=(1-\alpha)(1+\alpha)^{2}, \quad z(0)=-1-\alpha,
$$

and hence

$$
R\left(q_{-}(0, \alpha)\right)=q_{+}\left(T_{+}(\alpha), \alpha\right)=\left(\begin{array}{c}
0 \\
(1+\alpha) \sqrt{1-\alpha}
\end{array}\right) .
$$

We observe that $T_{+}^{0}$ is the first positive time such that $x\left(T_{+}^{0}\right)=0$ where $x(t)=z_{+}(t, 1)$ is the solution of

$$
\dot{x}^{2}+(x+2) x^{2}=1, \quad x(0)=-1,
$$

hence

$$
T_{+}^{0}=\int_{-1}^{0} \frac{d x}{\sqrt{1-x^{2}(x+2)}} \simeq 1.88292
$$

More related results are derived in the Appendix.

Then equations (6.2) have to be changed to

$$
\begin{aligned}
& R\left(q_{-}(0,0)\right)=q_{-}\left(-\frac{3}{2} \pi, 0\right), \\
& \frac{\partial}{\partial \alpha}\left[R\left(q_{-}(0, \alpha)\right)-q_{-}\left(-\frac{3}{2} \pi, \alpha\right)\right]_{\alpha=0} \neq 0 .
\end{aligned}
$$


But

$$
R\left(q_{-}(0, \alpha)\right)-q_{-}\left(-\frac{3}{2} \pi, \alpha\right)=(1+\alpha)\left(\begin{array}{c}
0 \\
\sqrt{1-\alpha}-1
\end{array}\right)
$$

and (6.8) easily follows. Now we compute the variational equation and the PoincaréMelnikov function. From (6.6) and $q_{-}(0, \alpha)=\left(\begin{array}{c}-(\alpha+1) \\ 0\end{array}\right)$, it follows that we can take

$$
R\left(x_{1}, x_{2}\right)=\left(\begin{array}{c}
0 \\
-x_{1} \sqrt{x_{1}+2}
\end{array}\right)
$$

from which we get

$$
R^{\prime}\left(q_{-}(0,0)\right)=\left(\begin{array}{cc}
0 & 0 \\
-\frac{1}{2} & 0
\end{array}\right)
$$

Note $\ddot{x}+2 x+\frac{3}{2} x^{2}=0$ has a homoclinic solution $-\frac{2}{3}\left(3 \tanh \left[\frac{t}{\sqrt{2}}\right]^{2}-1\right)$, so the solution $q_{+}(t, \alpha)$ is a part of a periodic solution inside of $\bar{\Omega}_{+}$bounded by the homoclinic one (see Figure 1 ). Then, since in a neighborhood of $q_{-}\left(-\frac{3}{2} \pi, 0\right)=\left(\begin{array}{l}0 \\ 1\end{array}\right)$ we have $G\left(x_{1}, x_{2}\right)=-x_{1}$ we get

$$
G^{\prime}\left(q_{-}\left(-\frac{3}{2} \pi, 0\right)\right)=\left(\begin{array}{c}
-1 \\
0
\end{array}\right)
$$

Finally, since the equations on $\Omega_{+}$can be written as

$$
\left\{\begin{array}{l}
\dot{x}_{1}=x_{2} \\
\dot{x}_{2}=-2 x_{1}-\frac{3}{2} x_{1}^{2}
\end{array}\right.
$$

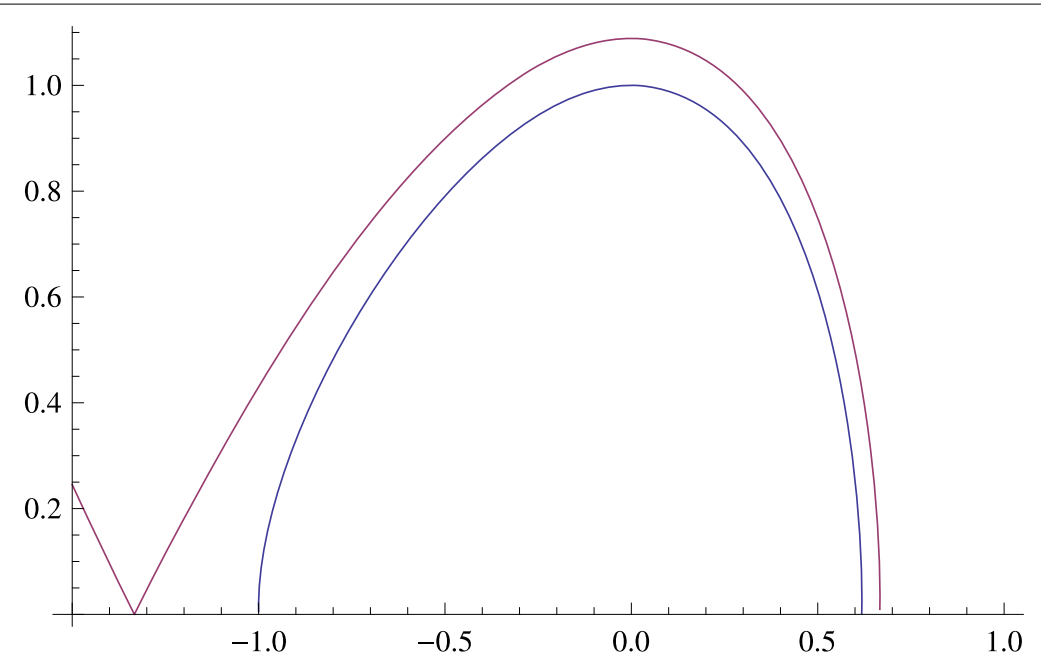

Figure 1 The upper parts of homoclinic and periodic orbits of $\ddot{x}+2 x+\frac{3}{2} x^{2}=0$. 
we get

$$
f_{+}\left(q_{+}\left(T_{+}(\alpha), \alpha\right)\right)=f_{+}(0,(1+\alpha) \sqrt{1-\alpha})=\left(\begin{array}{c}
(1+\alpha) \sqrt{1-\alpha} \\
0
\end{array}\right) .
$$

Putting all together we see that the adjoint variational system reads:

$$
\left\{\begin{array} { l } 
{ \ddot { w } + w = 0 , } \\
{ ( \begin{array} { c } 
{ - 1 } \\
{ 0 }
\end{array} ) [ ( \begin{array} { c } 
{ - \dot { w } ( 0 ) } \\
{ w ( 0 ) }
\end{array} ) - ( \begin{array} { c } 
{ - \frac { 1 } { 2 } \psi ^ { \prime \prime } } \\
{ 0 }
\end{array} ) ] = 0 , } \\
{ ( \begin{array} { c } 
{ - \dot { w } ( - \frac { 3 } { 2 } \pi ) } \\
{ w ( - \frac { 3 } { 2 } \pi ) }
\end{array} ) = ( \begin{array} { l } 
{ \psi ^ { \prime } } \\
{ \psi ^ { \prime \prime } }
\end{array} ) + \psi _ { 1 } ( \begin{array} { c } 
{ - 1 } \\
{ 0 }
\end{array} ) , } \\
{ ( \begin{array} { l } 
{ \psi ^ { \prime } } \\
{ \psi ^ { \prime \prime } }
\end{array} ) \cdot ( \begin{array} { l } 
{ 1 } \\
{ 0 }
\end{array} ) = 0 }
\end{array} \Leftrightarrow \left\{\begin{array}{l}
\ddot{w}+w=0, \\
\dot{w}(0)=\frac{1}{2} \psi^{\prime \prime}, \\
w\left(-\frac{3}{2} \pi\right)=\psi^{\prime \prime}, \\
\dot{w}\left(-\frac{3}{2} \pi\right)=-\psi^{\prime}+\psi_{1}, \\
\psi^{\prime}=0 .
\end{array}\right.\right.
$$

The first three equations give the boundary value problem

$$
\ddot{w}+w=0, \quad 2 \dot{w}(0)-w\left(-\frac{3}{2} \pi\right)=0
$$

possessing the unique solution (up to a multiplicative constant) $w(t)=\cos t$ which gives

$$
\psi^{\prime}=0, \quad \psi^{\prime \prime}=0, \quad \psi_{1}=-1
$$

and, since $g_{+}(t, x, \varepsilon)=0$, the Poincaré-Melnikov function is

$$
\mathcal{M}(\tau)=\int_{-\frac{3}{2} \pi}^{0} g_{-}(t+\tau,-\cos t, \sin t, 0) \cos t d t
$$

We conclude with the following.

Corollary 6.2 Let $T_{+}^{0}$ be as in equation (6.7), $g_{-}(t, x, \dot{x}, \varepsilon)$ be a $\left(\pi+\varepsilon T_{+}^{0}\right)$-periodic, $C^{2}$ function and suppose that the function (6.9) has a simple zero at $\tau=0$. Then, for $\varepsilon>0$, sufficiently small the singularly perturbed system

$$
\begin{cases}\varepsilon^{2} \ddot{x}+2 x+\frac{3}{2} x^{2}=0 & \text { if } x<0 \text { and } \dot{x}>0 \\ \ddot{x}+x=\varepsilon g_{-}(t, x, \dot{x}, \varepsilon) & \text { elsewhere }\end{cases}
$$

has a $\left(\pi+\varepsilon T_{+}^{0}\right)$-periodic solution orbitally near the set $\left\{(-\cos t, \sin t) \mid-\frac{3}{2} \pi \leq t \leq 0\right\} \cup$ $\left\{\left(z_{+}(t, 0), \dot{z}_{+}(t, 0)\right) \mid 0 \leq t \leq T_{+}^{0}\right\}$.

As an example of the second situation, we consider the case where $f_{+}(x)=f_{-}(x)=-x$, $\Omega_{-}=\{(x, \dot{x}) \mid x<0\}, \Omega_{+}=\{(x, \dot{x}) \mid x>0\}$, i.e., we take

$$
\begin{cases}\varepsilon^{2} \ddot{x}+x=0 & \text { if } x>0, \\ \ddot{x}+x=\varepsilon g_{-}(t, x, \dot{x}, \varepsilon) & \text { if } x<0\end{cases}
$$

where $g_{-}(t, x, \dot{x}, \varepsilon)$ is a $(1+\varepsilon) \pi$-periodic, $C^{2}$ function. Since

$$
q_{+}(t, \alpha)=\left(\alpha+\alpha_{0}\right)\left(\begin{array}{c}
\sin t \\
\cos t
\end{array}\right), \quad 0 \leq t \leq \pi
$$


we get $\varphi(\alpha)=-\alpha-\alpha_{0}$ for any $\alpha$ in a neighborhood of $\alpha=0$ and $\alpha_{0}>0$. Hence, we are in the degenerate case considered in Section 5 . The adjoint variational equation along $q_{-}(t, \alpha)$ reads now

$$
\left\{\begin{array}{l}
\ddot{w}+w=0, \\
w(0)+w(-\pi)=0, \\
\psi^{\prime \prime}=w(-\pi), \\
-\left(\alpha+\alpha_{0}\right) \psi^{\prime}=0, \\
\psi_{1}=-\dot{w}(-\pi)-\psi^{\prime} .
\end{array}\right.
$$

The first two equations have the two-dimensional family of solutions $w(t)=c \cos \left(t+t_{0}\right)$. We take the two independent solutions: $w_{1}(t)=\cos t$ and $w_{2}(t)=\sin t$ with the corresponding vectors:

$$
\begin{array}{lll}
\psi_{1}^{\prime \prime}=-1, & \psi_{1}^{\prime}=0, & \psi_{1}^{(1)}=0, \\
\psi_{2}^{\prime \prime}=0, & \psi_{2}^{\prime}=0, & \psi_{1}^{(2)}=1 .
\end{array}
$$

With $g_{+}(t, x, \dot{x}, \varepsilon)=0$ (which implies $R(\tau ; \xi, \varepsilon)$ is independent of $\varepsilon$ ) the Poincaré-Melnikov vector is then

$$
\mathcal{M}(\alpha, \tau)=\left(\begin{array}{l}
\int_{-\pi}^{0} g_{-}(t+\tau, \alpha \sin t, \alpha \cos t, 0) \cos t d t \\
\int_{-\pi}^{0} g_{-}(t+\tau, \alpha \sin t, \alpha \cos t, 0) \sin t d t
\end{array}\right) .
$$

Then we obtain the following corollary.

Corollary 6.3 $\operatorname{Letg}_{-}(t, x, \dot{x}, \varepsilon)$ be a $(1+\varepsilon) \pi$-periodic, $C^{2}$ function and suppose that $\mathcal{M}(\alpha, \tau)$ has a simple zero at $\alpha=\alpha_{0}, \tau=0$. Then the singularly perturbed system (6.10) has a $(1+$ $\varepsilon) \pi$-periodic solution orbitally near the $\operatorname{set}\{(\sin t, \cos t) \mid-\pi \leq t \leq \pi\}$.

\section{Appendix}

\section{A.1 Further properties of the solution of (6.5)}

From the identity

$$
\dot{z}_{+}^{2}(t, \alpha)+\left(z_{+}(t, \alpha)+2\right) z_{+}^{2}(t, \alpha)=(1-\alpha)(1+\alpha)^{2}
$$

we derive

$$
\frac{\dot{z}_{+}^{2}(t, \alpha)}{(1-\alpha)(1+\alpha)^{2}-\left(z_{+}(t, \alpha)+2\right) z_{+}^{2}(t, \alpha)}=1 \text {. }
$$

Note that

$$
\begin{aligned}
(1-\alpha)(1+\alpha)^{2}-(x+2) x^{2} & =-(1+\alpha+x)\left(-1+\alpha^{2}+x-\alpha x+x^{2}\right) \\
& =\left(A_{\alpha}-x\right)\left(x-B_{\alpha}\right)\left(x-C_{\alpha}\right),
\end{aligned}
$$


where

$$
\begin{aligned}
& A_{\alpha}=\frac{-1+\alpha+\sqrt{5-2 \alpha-3 \alpha^{2}}}{2}, \quad B_{\alpha}=-1-\alpha, \\
& C_{\alpha}=\frac{-1+\alpha-\sqrt{5-2 \alpha-3 \alpha^{2}}}{2}
\end{aligned}
$$

and, for $\alpha$ sufficiently small (in fact for $\alpha \in\left(-1, \frac{1}{3}\right)$ )

$$
A_{\alpha}>0>B_{\alpha}>C_{\alpha} .
$$

Using formula 3.131 .5 in [16, p.254] we know that, for any $A \geq u>B>C$ :

$$
\int_{B}^{u} \frac{d x}{\sqrt{(A-x)(x-B)(x-C)}}=\frac{2 F(\kappa, p)}{\sqrt{A-C}},
$$

where

$$
\kappa:=\arcsin \sqrt{\frac{(A-C)(u-B)}{(A-B)(u-C)}}, \quad p:=\sqrt{\frac{A-B}{A-C}}
$$

and $F$ is the elliptic integral of the first kind.

Next note $-1-\alpha \leq z_{+}(t, \alpha) \leq 0, \dot{z}_{+}(t, \alpha) \geq 0$ for $t \in\left[0, T_{+}(\alpha)\right]$ and $z_{+}(0, \alpha)=-1-\alpha$, $z_{+}\left(T_{+}(\alpha), \alpha\right)=0$. Hence, (7.1) gives

$$
\begin{aligned}
T_{+}(\alpha) & =\int_{0}^{T_{+}(\alpha)} d t=\int_{0}^{T_{+}(\alpha)} \frac{\dot{z}_{+}(t, \alpha)}{\sqrt{(1-\alpha)(1+\alpha)^{2}-\left(z_{+}(t, \alpha)+2\right) z_{+}^{2}(t, \alpha)}} \\
& =\int_{B_{\alpha}}^{0} \frac{d x}{\sqrt{\left(A_{\alpha}-x\right)\left(x-B_{\alpha}\right)\left(x-C_{\alpha}\right)}}=\frac{2 F\left(\kappa_{\alpha}, p_{\alpha}\right)}{\sqrt{A_{\alpha}-C_{\alpha}}}
\end{aligned}
$$

where

$$
\begin{aligned}
& \kappa_{\alpha}=\arcsin \left[\sqrt{\frac{10+6 \alpha}{5+3 \alpha+3 \sqrt{5-2 \alpha-3 \alpha^{2}}}}\right] \\
& p_{\alpha}=\sqrt{\frac{1+3 \alpha+\sqrt{5-2 \alpha-3 \alpha^{2}}}{2 \sqrt{(1-\alpha)(5+3 \alpha)}} .}
\end{aligned}
$$

So,

$$
T_{+}(\alpha)=\frac{2 F\left(\kappa_{\alpha}, p_{\alpha}\right)}{\sqrt[4]{5-2 \alpha-3 \alpha^{2}}} .
$$

We are interested in $\alpha=0$. Then

$$
\kappa_{0}=\arcsin \left[\sqrt{\frac{10}{5+3 \sqrt{5}}}\right], \quad p_{0}=\frac{\sqrt{1+\sqrt{5}}}{\sqrt{2} \sqrt[4]{5}},
$$


and hence

$$
T_{+}(0)=\frac{2 F\left(\arcsin \left[\sqrt{\frac{10}{5+3 \sqrt{5}}}\right], \frac{\sqrt{1+\sqrt{5}}}{\sqrt{2} \sqrt[4]{5}}\right)}{\sqrt[4]{5}} \doteq 1.88292
$$

On the other hand, by (6.7), we directly verify that $T_{+}(0) \doteq 1.88292$ by a numerical integration. But we derived (7.2) to get an explicit formula for $T_{+}(0)$ and in general for $T_{+}(\alpha)$. Furthermore, the above computations also give

$$
t=\int_{B_{\alpha}}^{z_{+}(t, \alpha)} \frac{d x}{\sqrt{\left(A_{\alpha}-x\right)\left(x-B_{\alpha}\right)\left(x-C_{\alpha}\right)}}=\frac{2 F\left(\kappa_{\alpha}(t), p_{\alpha}\right)}{\sqrt[4]{5-2 \alpha-3 \alpha^{2}}}
$$

for any $0 \leq t \leq T_{+}(\alpha)$ and

$$
\begin{aligned}
& \kappa_{\alpha}(t) \\
& \quad=\arcsin \left[2 \sqrt{\frac{\sqrt{(1-\alpha)(5+3 \alpha)}\left(1+\alpha+z_{+}(t, \alpha)\right)}{\left(1+3 \alpha+\sqrt{5-2 \alpha-3 \alpha^{2}}\right)\left(1-\alpha+\sqrt{5-2 \alpha-3 \alpha^{2}}+2 z_{+}(t, \alpha)\right)}}\right] .
\end{aligned}
$$

Solving (7.3), we obtain

$$
\kappa_{\alpha}(t)=\operatorname{am}\left(\sqrt[4]{5-2 \alpha-3 \alpha^{2}} \frac{t}{2}, p_{\alpha}\right)
$$

where am is the Jacobi amplitude function. Solving (7.4), we obtain

$$
\begin{aligned}
& z_{+}(t, \alpha) \\
& \quad=\frac{(1+\alpha)\left(-3+\sqrt{5-2 \alpha-3 \alpha^{2}}-3 \alpha\left(-1+H_{\alpha}(t)\right)+\left(3+\sqrt{5-2 \alpha-3 \alpha^{2}}\right) H_{\alpha}(t)\right)}{1-\sqrt{5-2 \alpha-3 \alpha^{2}}-3 \alpha\left(-1+H_{\alpha}(t)\right)-H_{\alpha}(t)-\sqrt{5-2 \alpha-3 \alpha^{2}} H_{\alpha}(t)}
\end{aligned}
$$

for

$$
H_{\alpha}(t):=\cos ^{2}\left(\kappa_{\alpha}(t)\right)=\operatorname{cn}^{2}\left(\sqrt[4]{5-2 \alpha-3 \alpha^{2}} \frac{t}{2}, p_{\alpha}\right)
$$

where $\mathrm{cn}$ is the Jacobi elliptic function. Formulas (7.5) and (7.6) give explicit solution $z_{+}(t, \alpha)$. For $\alpha=0$, we derive

$$
z_{+}(t, 0)=\frac{-3+\sqrt{5}+(3+\sqrt{5}) \operatorname{cn}\left(\sqrt[4]{5} \frac{t}{2}, \frac{\sqrt{1+\sqrt{5}}}{\sqrt{2} \sqrt[4]{5}}\right)^{2}}{1-\sqrt{5}-(1+\sqrt{5}) \operatorname{cn}\left(\sqrt[4]{5} \frac{t}{2}, \frac{\sqrt{1+\sqrt{5}}}{\sqrt{2} \sqrt[4]{5}}\right)^{2}}
$$

We can also compute the Taylor series of (7.7) integrating by series the equation $\ddot{x}+2 x+$ $\frac{3}{2} x^{2}=0$ with $x(0)=-1, \dot{x}(0)=0$. Setting

$$
x(t)=-1+\sum_{n=2}^{\infty} \frac{a_{n}}{n !} t^{n}
$$


we see that the following recurrence condition holds:

$$
\frac{a_{n+2}}{n !}+2 \frac{a_{n}}{n !}+\frac{3}{2} \sum_{h=0}^{n} \frac{a_{n-h}}{(n-h) !} \frac{a_{h}}{h !}=0 \quad \Leftrightarrow \quad a_{n+2}+2 a_{n}+\frac{3}{2} \sum_{h=0}^{n}\left(\begin{array}{l}
n \\
h
\end{array}\right) a_{n-h} a_{h}=0,
$$

where we set $\left(\begin{array}{l}0 \\ 0\end{array}\right)=1$ and $a_{0}=-1, a_{1}=0$. Since $a_{1}=0$, we see by the induction that $a_{2 k+1}=0$ for any $k \in \mathbb{N}$ (note that in the product $a_{h} a_{2 k+1-h}$ one of the two indexes is odd). So,

$$
x(t)=-1+\sum_{n=1}^{\infty} a_{2 n} \frac{t^{2 n}}{(2 n) !}
$$

and

$$
a_{2(n+1)}+2 a_{2 n}+\frac{3}{2} \sum_{h=0}^{n}\left(\begin{array}{l}
2 n \\
2 h
\end{array}\right) a_{2(n-h)} a_{2 h}=0 .
$$

For the first few indexes, we get

$$
\begin{aligned}
& a_{2}=a_{4}=\frac{1}{2}, \quad a_{6}=a_{4}-9 a_{2}^{2}=-\frac{7}{4}, \quad a_{8}=a_{6}-45 a_{2} a_{4}=-13, \\
& a_{10}=a_{8}-84 a_{2} a_{6}-105 a_{4}^{2}=\frac{137}{4} a_{12}=a_{10}-135 a_{2} a_{8}-630 a_{4} a_{6}=1463
\end{aligned}
$$

so that:

$$
z_{+}(t, 0)=-1+\frac{1}{2 \cdot 2 !} t^{2}+\frac{1}{2 \cdot 4 !} t^{4}-\frac{7}{4 \cdot 6 !} t^{6}-\frac{13}{8 !} t^{8}+\frac{137}{4 \cdot 10 !} t^{10}+\frac{1463}{12 !} t^{12}+\cdots .
$$

On the other hand, using Mathematica, we can expand (7.7) to get

$$
\begin{aligned}
z_{+}(t, 0)= & -1+\frac{t^{2}}{4}+\frac{t^{4}}{48}-\frac{7 t^{6}}{2,880}-\frac{13 t^{8}}{40,320}+\frac{137 t^{10}}{14,515,200}+\frac{19 t^{12}}{6,220,800} \\
& +\frac{2,531 t^{14}}{63,402,393,600}-\frac{82,291 t^{16}}{3,804,143,616,000}-\frac{179,107 t^{18}}{166,295,420,928,000} \\
& +\frac{1,972,291 t^{20}}{17,013,300,756,480,000}+\cdots,
\end{aligned}
$$

which coincides with our above analytical expansion.

\section{A.2 Proof of Theorem 3.2}

Here, we prove Theorem 3.2. We emphasize the fact that proof mainly follows the idea in [13, Theorem 4.1].

Proof of Theorem 3.2 The existence part is quite standard so we sketch it and give emphasis to the proof of invertibility of $D_{1} F(x(\varepsilon), \varepsilon)$ for $\varepsilon \neq 0$. Since $F(\xi(\mu), 0)=0$, we get $L(\mu) \xi^{\prime}(\mu)=0$ and, differentiating twice, $D_{1}^{2} F(\xi(\mu), 0)\left(\xi^{\prime}(\mu), \xi^{\prime}(\mu)\right)+L(\mu) \xi^{\prime \prime}(\mu)=0$. As a consequence, $\xi^{\prime}(\mu) \in \mathcal{N} L(\mu)$ and

$$
D_{1}^{2} F(\xi(\mu), 0)(v, w) \in \mathcal{R} L(\mu) \quad \text { for any } v, w \in \mathcal{N} L(\mu) .
$$


Let $\Pi(\mu): Y \rightarrow \mathcal{R} L(\mu)$ as in the statement of the theorem. We write $x=z+\xi(\mu)$, with $z \in \mathcal{N} L(\mu)^{\perp}$. Applying the Implicit Function Theorem to the map $(z, \mu, \varepsilon) \mapsto \Pi(\mu) F(z+$ $\xi(\mu), \varepsilon)$, we get the existence of a unique $C^{2}$-solution $z=z(\mu, \varepsilon) \in \mathcal{N} L(\mu)^{\perp}$ of the equation $\Pi(\mu) F(z+\xi(\mu), \varepsilon)=0$. From uniqueness, we obtain also

$$
z(\mu, 0)=0 .
$$

Next, differentiating the equality $\Pi(\mu) F(z(\mu, \varepsilon)+\xi(\mu), \varepsilon)=0$ with respect to $\mu$ and to $\varepsilon$ at $(\mu, 0)$, we get:

$$
\begin{aligned}
& \Pi(\mu) L(\mu)\left[z_{\mu}(\mu, 0)+\xi^{\prime}(\mu)\right]=0 \quad \Rightarrow \quad z_{\mu}(\mu, 0) \in \mathcal{N} L(\mu), \\
& \Pi(\mu)\left[L(\mu) z_{\varepsilon}(\mu, 0)+D_{2} F(\xi(\mu), 0)\right]=0 \quad \Rightarrow \quad L(\mu) z_{\varepsilon}(\mu, 0)=-\Pi(\mu) D_{2} F(\xi(\mu), 0) .
\end{aligned}
$$

Next, for $\varepsilon \neq 0$, equation $[\mathbb{I}-\Pi(\mu)] F(z(\mu, \varepsilon)+\xi(\mu), \varepsilon)=0$ is equivalent to $\varepsilon^{-1}[\mathbb{I}-\Pi(\mu)] \times$ $F(z(\mu, \varepsilon)+\xi(\mu), \varepsilon)=0$, but the l.h.s. tends, for $\varepsilon \rightarrow 0$ to $[\mathbb{I}-\Pi(\mu)] D_{2} F(\xi(\mu), 0)$ which gives the Poincaré-Melnikov condition. We conclude that, if the Poincaré-Melnikov condition is satisfied, for $\varepsilon \neq 0$ (small) there exists a unique solution of equation $F(x, \varepsilon)=0, x=x(\varepsilon)=$ $z(\mu(\varepsilon), \varepsilon)+\xi(\mu(\varepsilon))$, with $\mu(0)=0$.

Now we prove the invertibility of $D_{1} F(x(\varepsilon), \varepsilon)$. Since $D_{1} F(x(\varepsilon), \varepsilon)$ is Fredholm with index zero, it is enough to prove that equation $D_{1} F(x(\varepsilon), \varepsilon) z=0$ has, for $\varepsilon \neq 0$, the unique solution $z=0$. Although $\mathcal{F}(z, \varepsilon):=D_{1} F(x(\varepsilon), \varepsilon) z$ is only $C^{1}$ with respect to $\varepsilon$, it is linear in $z$. Thus, we can still apply the existence and uniqueness argument given above. Of course, $\mathcal{F}(z, 0)$ vanishes on the linear subspace $\widetilde{\mathcal{M}}:=\{z \in \mathcal{N} L(0)\}$, and clearly $\mathcal{N} D_{1} \mathcal{F}(z, 0)=\{z \in \mathcal{N} L(0)\}$. Next $\mathcal{R} D_{1} \mathcal{F}(z, 0)=\mathcal{R} L(0)$ so that $\widetilde{\Pi}(\mu)=\Pi(0)$. Thus, from the existence and uniqueness result it follows that $\mathcal{N} D_{1} F(x(\varepsilon), \varepsilon)=\{0\}$ if the following condition is satisfied:

$$
z \in \mathcal{N} L(0) \quad \text { and } \quad[\mathbb{I}-\Pi(0)]\left[D_{1}^{2} F(0,0) x^{\prime}(0)+D_{1} D_{2} F(0,0)\right] z=0 \quad \Rightarrow \quad z=0 .
$$

On account of $x(\varepsilon)=z(\mu(\varepsilon), \varepsilon)+\xi(\mu(\varepsilon))$ we are led to look at the solutions of

$$
[\mathbb{I}-\Pi(0)]\left[D_{1}^{2} F(0,0)\left(z_{\mu}(0,0) \mu^{\prime}(0)+z_{\varepsilon}(0,0)+\xi^{\prime}(0) \mu^{\prime}(0)\right)+D_{1} D_{2} F(0,0)\right] z=0
$$

with $z \in \mathcal{N} L(0)$. From the previous remarks, we get:

$$
D_{1}^{2} F(0,0)\left(z_{\mu}(0,0) \mu^{\prime}(0), z\right) \in \mathcal{R} L(0) \quad \text { and } \quad D_{1}^{2} F(0,0)\left(\xi^{\prime}(0) \mu^{\prime}(0), z\right) \in \mathcal{R} L(0)
$$

for any $z \in \mathcal{N} L(0)$, since $z_{\mu}(0,0) \mu^{\prime}(0), \xi^{\prime}(0) \mu^{\prime}(0) \in \mathcal{N} L(0)$. So, the claim to be proved is

$$
[\mathbb{I}-\Pi(0)]\left[D_{1}^{2} F(0,0) z_{\varepsilon}(0,0)+D_{1} D_{2} F(0,0)\right] \xi^{\prime}(0) \neq 0,
$$

where we have replaced $z$ with $\xi^{\prime}(0)$ since $\mathcal{N} L(0)=\operatorname{span}\left\{\xi^{\prime}(0)\right\}$. Now we differentiate the equality

$$
L(\mu) z_{\varepsilon}(\mu, 0)=-\Pi(\mu) D_{2} F(\xi(\mu), 0)=\mathcal{M}(\mu)-D_{2} F(\xi(\mu), 0)
$$


with respect to $\mu$ at $\mu=0$ to get

$$
D_{1}^{2} F(0,0) z_{\varepsilon}(0,0) \xi^{\prime}(0)+L(0) z_{\varepsilon \varepsilon}(0,0)=\mathcal{M}^{\prime}(0)-D_{1} D_{2} F(0,0) \xi^{\prime}(0) .
$$

Hence,

$$
[\mathbb{I}-\Pi(0)]\left[D_{1}^{2} F(0,0) z_{\varepsilon}(0,0)+D_{1} D_{2} F(0,0)\right] \xi^{\prime}(0)=[\mathbb{I}-\Pi(0)] \mathcal{M}^{\prime}(0) \neq 0
$$

since, from $[\mathbb{I}-\Pi(\mu)] \mathcal{M}(\mu)=\mathcal{M}(\mu)$ and $\mathcal{M}(0)=0$ we get $[\mathbb{I}-\Pi(0)] \mathcal{M}^{\prime}(0)=\mathcal{M}^{\prime}(0)$. The proof of Theorem 3.2 is complete.

\section{Competing interests}

The authors declare that they have no competing interests.

\section{Authors' contributions}

The work presented here was carried out in collaboration between the authors. The authors contributed to every part of this study equally and read and approved the final version of the manuscript.

\section{Author details}

'Dipartimento di Ingegneria Industriale e Scienze Matematiche, Marche Polytecnic University, Ancona, 60100, Italy.

${ }^{2}$ Department of Mathematical Analysis and Numerical Mathematics, Faculty of Mathematics, Physics and Informatics,

Comenius University, Mlynská dolina, Bratislava, 842 48, Slovakia.

\section{Acknowledgements}

This article is dedicated to Professor Jean Mawhin on the occasion of his 70th birthday.

BF is partially supported by GNAMPA-CNR and MURST-group 'Equazioni differenziali ordinarie e applicazioni' (Italy). MF is partially supported by the grant APVV-0134-10 and Marche Polytechnic University, Ancona (Italy).

Received: 14 October 2012 Accepted: 21 March 2013 Published: 3 April 2013

\section{References}

1. Bernardo, M, Budd, C, Champneys, AR, Kowalczyk, P: Piecewise-smooth Dynamical Systems: Theory and Applications. Springer, London (2008)

2. Brogliato, B: Nonsmooth Impact Mechanics. Springer, Berlin (1996)

3. Chua, LO, Komuro, M, Matsumoto, T: The double scroll family. IEEE Trans. Circuits Syst. 33, 1073-1118 (1986)

4. Kunze, M, Küpper, T: Non-smooth dynamical systems: an overview. In: Ergodic Theory, Analysis and Efficient Simulation of Dynamical Systems, pp. 431-452. Springer, Berlin (2001)

5. Kuznetsov, YA, Rinaldi, S, Gragnani, A: One-parametric bifurcations in planar Filippov systems. Int. J. Bifurc. Chaos 13 2157-2188 (2003)

6. Leine, RI, Van Campen, DH, Van de Vrande, BL: Bifurcations in nonlinear discontinuous systems. Nonlinear Dyn. 23 105-164 (2003)

7. Awrejcewicz, J, Fečkan, M, Olejnik, P: Bifurcations of planar sliding homoclinics. Math. Probl. Eng. 2006, 1-13 (2006)

8. Teixeira, MA, Silva, PR: Regularization and singular perturbation techniques for non-smooth systems. Physica D 241 , 1948-1955 (2012)

9. Fenichel, N: Geometric singular perturbation theory for ordinary differential equations. J. Differ. Equ. 31, 53-98 (1979)

10. Jones, C: Geometric singular perturbation theory. In: Dynamical Systems, C.I.M.E. Lectures, Montecatini Terme, 1994. Lect. Notes Math., vol. 1609. Springer, Heidelberg (1995)

11. Ivanov, A: Bifurcations in impact systems. Chaos Solitons Fractals 7, 1615-1634 (1996)

12. Makarenkov, $\mathrm{O}$, Verhulst, F: Bifurcation of asymptotically stable periodic solutions in nearly impact oscillators. Preprint

13. Palmer, KJ: Exponential dichotomies and transversal homoclinic points. J. Differ. Equ. 55, 225-256 (1984)

14. Battelli, F, Fečkan, M: Chaos in forced impact systems. Discrete Contin. Dyn. Syst., Ser. S 6, 861-890 (2013)

15. Gaines, E, Mawhin, J: Coincidence Degree, and Nonlinear Differential Equations. Springer, Berlin (1977)

16. Gradshteyn, IS, Ryzhik, IM: Table of Integrals, Series, and Products. Academic Press, Boston (2007)

doi:10.1186/1687-2770-2013-71

Cite this article as: Battelli and Fečkan: Fast-slow dynamical approximation of forced impact systems near periodic solutions. Boundary Value Problems 2013 2013:71 